2000 MRS Fall Meeting Offers Smorgasbord of Materials Research Topics

The 2000 Materials Research Society Fall Meeting was held in Boston November 26-December 1 at the Hynes Convention Center and the Sheraton Boston Hotel. Chaired by Sungho Jin (Bell Labs/Lucent Technologies), Antonios G. Mikos (Rice University), David J. Srolovitz (Princeton University), and Bernd Stritzker (Universität Augsburg), the meeting hosted 40 technical symposia categorized into eight clusters, in addition to overviews presented in Symposium X: Frontiers of Materials Research, for the nonspecialist. The thread of nanotechnology ran through a number of symposia.

Special events included the plenary session, which featured a talk on soft matter by Nobel laureate Pierre-Gilles de Gennes (Collège de France, ESPCI). The awards ceremony, held on November 29, honored George Whitesides (Harvard) with the Von Hippel Award, Anthony Evans (Princeton) with the David Turnbull Lectureship, and Dieter Gruen (ANL) and Samuel Stupp (Northwestern) each with an MRS Medal. Graduate student finalists received Gold or Silver awards. "Research Tools Seminars" debuted at the meeting in conjunction with the equipment exhibit. A number of presentations in the seminar focused on the scientific bases for and practical applications of commercially available state-of-the-art tools for materials research. The equipment exhibit featured more than 225 exhibitors from around the world, displaying a range of equipment, services, products, publications, and software. See Sidebars covering these and other events.

\section{Technical Sessions}

\section{Organic and Biomaterials}

To realize smart packages and dynamic information surfaces, M. Berggren (ACREO, Norrkoping, Sweden) has developed active functions on the surface of paper. Presenting results from the PAELLA project (paper electronics lowcost applications) in Symposium JJ, Berggren described conjugated polymers and organic molecules as the key materials. This "dynamic image" technology is fabricated on the paper surface through printing and reel-to-reel deposition processes that offer low-cost and largearea production. The work combines multicolor pigments and electronic updating technology on paper. Smart packages, wireless communications on paper surfaces, and active displays on walls or billboards are among the possible applications.

By using bone growth as a guide, C.M. Dry (University of Illinois School of Architecture) has developed composites of polymers with cement that have unique toughness and strength. As described in Symposium KK, hollow porous-walled structural fibers are woven and placed first. Fiber delivery systems, both straight and spiral-shaped tubules, are required to evenly distribute the liquid chemical initiator to all portions of the powder matrix of monomer and cement. Chemicals are released through these fibers to react with each other in a predetermined order and place. The polymer reacts and forms first, yielding an effluent with which the second material, the cement, reacts and follows the form of the polymer. In effect, the polymer acts as a template. The second reaction, the hydration of the cement, drives the first (i.e., the polymerization) by taking up the effluent and supplying heat for the endothermic polymerization reaction. The cement takes on the form of the polymer as a template. The resulting chemically bonded composite exhibits ductile rather than brittle failure. The advantages of this composite-forming process are that all of the reaction products are utilized in the process, no mixing or heating is required, the fibers are intimately bonded to the matrix, and the composite is less prone to catastrophic failure.

Researchers at the Massachusetts Institute of Technology are using polymer scaffolds (structural templates) and bioreactors to grow new cartilage from cell cultures in vitro. In Symposium LL, G. Vunjak-Novakovik (MIT) reported on the use of polyglycolic acid polymers to form a mesh of $13-\mu \mathrm{m}$ fibers with $97 \%$ porosity to act as a scaffold to support tissue growth. By seeding the scaffolds with cartilage cells and growing them in a rotating bioreactor, functional cartilage samples were obtained after seven months.

Peter X. Ma described work being done at the University of Michigan to study the diffusion properties of ionically crosslinked alginate hydrogels. These hydrogels are hydrophilic and contain large amounts of water, similar to natural tissues; they can be molded to a desired shape, and they are injectable. This makes them ideal candidates for use as scaffolds (templates) for soft-tissue growth.

Researchers at Rice University, the M.D. Anderson Cancer Center in Houston, Texas, and the Mayo Clinic envision a biodegradable-polymer scaffold that could be injected into bone defects to promote hard-tissue repair. R.G. Payne (Rice) reported that by encapsulating rat marrow stromal cells in gelatin before incorporation into a poly(propylene fumarate) polymer, the cells were able to survive despite the heat generated by the exothermic cross-linking reaction and the fact that some monomers involved are cytotoxic.

By performing several thousand nanoindentation tests on a cross section of a human molar, A.B. Mann (Johns Hopkins) and his co-workers were able to determine the variations in elastic modulus and hardness with depth below the surface of the tooth. The investigators identified a very thin layer at the surface where the enamel is very hard and strong. Scanning electron microscopy (SEM) revealed differences in the hydroxyapatite crystal structure that partly explain these results.

M.V. Sefton (Univ. of Toronto), in Symposium MM, described one approach to grow human heart-muscle cells outside the body, in a scaffold made from a degradable material in the shape of the heart. The resulting heart muscle, together with separately prepared valves and other components, could potentially be assembled into the final product. The organs would then be stored until needed. Already, blood vessels have been generated without growth factors. Spin-off technologies such as valves, cardiac patches, and vascular grafts are likely to be major benefits as a result of this research.

In the area of drug delivery, E.W. Merrill (MIT), one of the pioneers in the field, described work with two-phase poly(ethylene glycol)-polysiloxane networks. In Symposium NN, Merrill described the use of these materials as inserts under the lower eyelid for ophthalmic drug delivery. This would replace eyedrops and would be more effective, based on time release. Merrill described the delivery of drugs such as 
Timolol (an anti-glaucoma drug), ofloxacin, and prednisolone.

N.A. Peppas (Purdue) described the use of complexation-sensitive hydrogels for oral protein delivery, by using crosslinked microparticles of poly(methacrylic acid) and PEG containing insulin. The three-dimensional network formed has PEG grafts as cross-linking interconnects in a complexed state. The PEG grafts serve as a stabilizer for trapped proteins and peptides. The release of insulin is based on the $\mathrm{pH}$ of the surrounding material, making $\mathrm{pH}$ a convenient tool for releasing the insulin at the appropriate location. A.S. Hoffman (Univ. of Washington) described the design of $\mathrm{pH}$-sensitive polymers to enhance intracellular trafficking of peptides, proteins, ODNs, and DNA. He described a nonviral technique to deliver the drug to the nucleus of a cell, based on phase-separation as a function of $\mathrm{pH}$.

In the area of neurologic biomaterials, Symposium OO covered specific structure-property-function relationships of mid- to long-term implant materials that come in contact with the nervous system. P.A. Tresco (Utah Univ.) discussed bioreactive materials for the neural interface. To date, most available material is either inert or biologically silent. There is a need for more bioreactive materials that will bind astrocytes and meningeal cells to the surface and promote neurite growth. By coating polystyrene devices with the celladhesion molecule L1-a powerful promoter of neuron outgrowth-binding of astrocytes, meningeal cells, and cerebellar

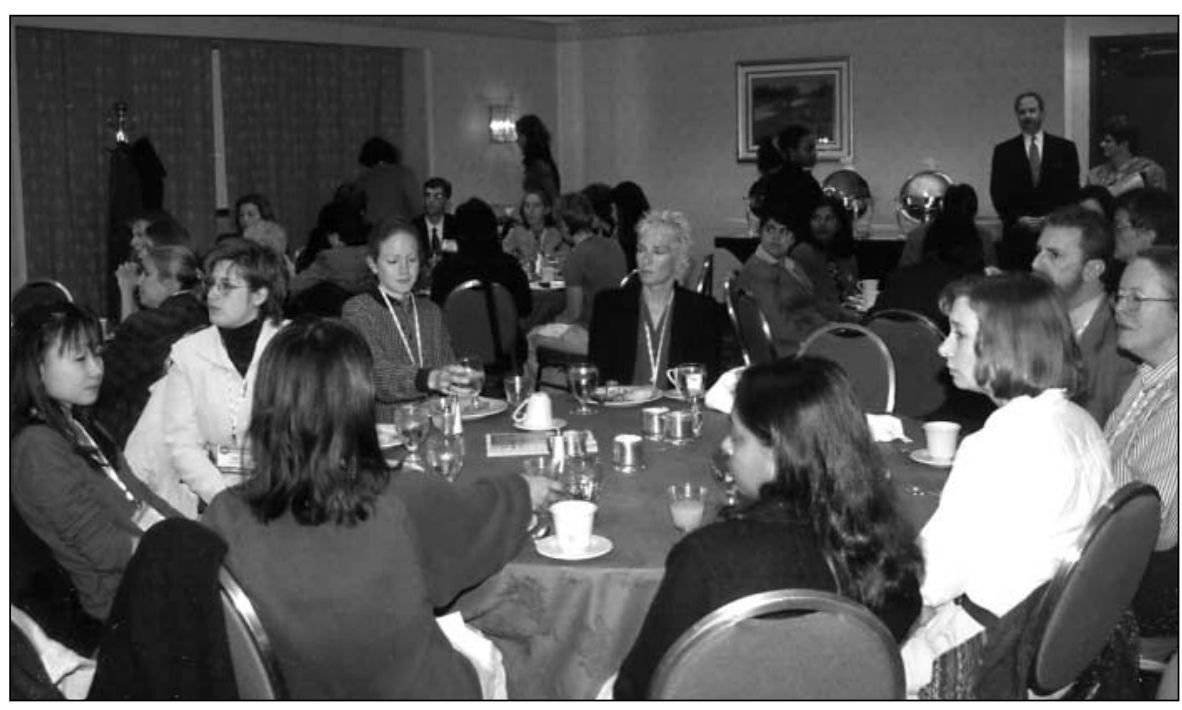

The MRS Outreach Subcommittee of the Public Affairs Committee sponsored a Women in Materials Science Breakfast. Among the major issues discussed was how to create an environment within industry and academia to support women materials researchers to be at their best. In addition, suggestions were given on how spouses may pursue their professional goals in parallel and how postdoctorates and researchers may negotiate within academia to obtain the resources they want to support their work.

cells is enhanced. Designing materials with the physical and mechanical properties of biological entities is also important. Reducing polypropylene filaments to a biologically compatible diameter of $35 \mathrm{~mm}$ has been shown to promote neuron attachment. Similarly, axonal guidance is enhanced when the stiffness of the biomaterial matches the intrinsic stiffness of neurons. By paying attention to these details, and others such as surface curvature and topography, biointeraction can be improved.

P.D. Dalton (Univ. of Toronto) reported on the development of a multicomponent tissue-engineered medical device that is designed to be an engineered nerve guide, a tissue-engineered scaffold, and a

\section{ACRONYM KEY}

1D: one-dimensional

2D: two-dimensional

3D: three-dimensional

4D: four-dimensional

AFM: atomic force microscopy

AFRL: Air Force Research Laboratory

ANL: Argonne National Laboratory

ARO: Army Research Office

Caltech: California Institute of Technology

CVD: chemical vapor deposition

CWRU: Case Western Reserve University

DARPA: Defense Advanced Research

Projects Agency

DoD: U.S. Department of Defense

DOE: U.S. Department of Energy

EELS: electron energy loss spectroscopy

ESEM: environmental scanning

electron microscopy

FET: field-effect transistor

GM: General Motors

HRSEM: high-resolution scanning electron microscopy

HRTEM: high-resolution transmission electron microscopy

HTS: high-temperature superconductor

IBAD: ion-beam-assisted deposition
IC: integrated circuit

IR: infrared

JRCAT: Joint Research Center for Atom Technology

LANL: Los Alamos National Laboratory

LAN: local-area network

LED: light-emitting diode

LEED: low-energy electron diffraction

MBE: molecular-beam epitaxy

MEMS: microelectromechanical system

MFM: magnetic force microscopy

MIT: Massachusetts Institute of Technology

MOCVD: metalorganic chemical vapor deposition

MOSFET: metal-oxide-semiconductor field-effect transistor

MOVPE: metalorganic vapor-phase epitaxy

MPI: Max-Planck-Institut

NCSU: North Carolina State University

NDE: nondestructive evaluation (of materials)

NIST: U.S. National Institute of Standards and Technology

NMR: nuclear magnetic resonance

NRL: U.S. Naval Research Laboratory

NSF: U.S. National Science Foundation

OLED: organic light-emitting diode
ONR: U.S. Office of Naval Research

ORNL: Oak Ridge National Laboratory

PMN-PT: $\mathrm{Pb}(\mathrm{Mg}, \mathrm{Nb}) \mathrm{O}_{3}-\mathrm{PbTiO}_{3}$

PSU: The Pennsylvania State University

PVD: physical vapor deposition

PZT: $\mathrm{Pb}(\mathrm{Zr}, \mathrm{Ti}) \mathrm{O}_{3}$

QD: quantum dot

R\&D: research and development

RAM: random-access memory

RF: radio frequency

RPI: Rensselaer Polytechnic Institute

SNL: Sandia National Laboratories

SOA: semiconductor optical amplifier

SPM: scanning probe microscopy

STEM: scanning transmission electron

microscopy

STM: scanning transmission microscopy

SUNY: State University of New York

SWNT: single-walled nanotube

TBC: thermal-barrier coating

TEM: transmission electron microscopy

TI: Texas Instruments

UCLA: University of California-Los Angeles

UCSB: University of California-Santa Barbara 
drug-delivery reservoir all in one. Its purpose is to contain and isolate therapy, and to provide a platform upon which future treatment schemes might be built. By combining poly(2-hydroxyl methacrylate) (PHEMA) with 25-wt\% methyl methacrylate (MMA), a tube with an elastic modulus that matches that of the spine was

\section{Technical Symposia}

\section{CLUSTER 1: Nano-/Microstructured Materials}

Symposium A: Nanotubes and Related Materials

Symposium B: Structure and Mechanical Properties of Nanophase Materials-Theory and Computer Simulations vs. Experiment

Symposium C: $\quad$ Anisotropic Nanoparticles-Synthesis, Characterization, and Applications Symposium D: Nonlithographic and Lithographic Methods for Nanofabrication-From Ultralarge-Scale Integration to Photonics to Molecular Electronics

Symposium E: $\quad$ Microphotonics-Materials, Physics, and Applications

Symposium F: Nano- and Microcrystalline Semiconductor Materials and Structures

CLUSTER 2: Semiconductors

Symposium G: GaN and Related Alloys

Symposium H: Silicon Carbide—Materials, Processing, and Devices

Symposium I: $\quad$ Semiconductor Spintronics-Physics, Materials, and Applications

Symposium J: Semiconductor Quantum Dots

\section{CLUSTER 3: Metals}

Symposium K: Quasicrystals

Symposium L: Supercooled Liquid, Bulk Glassy, and Nanocrystalline States of Alloys

Symposium M: Thermal Barrier Coatings

Symposium N: High-Temperature Ordered Intermetallic Alloys

\section{CLUSTER 4: Materials Processing \& Analysis}

Symposium O: Ion-Beam Synthesis and Processing of Advanced Materials

Symposium P: $\quad$ Growth, Evolution, and Properties of Surfaces, Thin Films, and SelfOrganized Structures

Symposium Q: Fundamentals of Nanoindentation and Nanotribology II

Symposium R: $\quad$ Microstructural Processes in Irradiated Materials

Symposium S: $\quad$ Applied Magnetic Field Effects on Materials Behavior

Symposium T: $\quad$ Dynamics in Confining Systems VI

Symposium U: Ultrafast Nonlinear Optical Phenomena

Symposium V: $\quad$ Low Vacuum SEM/ESEM in Materials Science-Wet SEM-The Liquid Frontier of Microscopy

CLUSTER 5: Defects, Mechanics, and Length Scales

Symposium W: The Limits of Strength in Theory and Practice

Symposium Y: Influences of Interface and Dislocation Behavior on Microstructure Evolution

Symposium Z: Multiscale Materials Modeling

Symposium AA: Structure-Property Relationships of Oxide Surfaces and Internal Interfaces

CLUSTER 6: Device and Functional Materials

Symposium BB: Characterization and Modeling of Domain Microstructures in Materials Symposium CC: Ferroelectric Thin Films IX

Symposium DD: Materials Issues for Tunable RF and Microwave Devices II

Symposium EE: Materials Science of Microelectromechanical System (MEMS) Devices III

CLUSTER 7: Inorganic Materials

Symposium FF: Materials Science of High-Performance Concrete

Symposium GG: Solid State Chemistry of Inorganic Materials

Symposium HH: Advanced Catalytic Materials-2000

Symposium II: High Temperature Superconductors-Crystal Chemistry, Processing, and Properties

CLUSTER 8: Organic and Biomaterials

Symposium JJ: Organic Electronic and Photonic Materials and Devices

Symposium KK: Filled and Nanocomposite Polymer Materials

Symposium LL: Orthopedic/Dental Biomaterials

Symposium MM: Cardiovascular Biomaterials

Symposium NN: Biomaterials for Drug Delivery

Symposium OO: Neurologic Biomaterials

\section{CLUSTER 9: GENERAL}

Symposium X: Frontiers of Materials Research

obtained. The fabrication of multilayer tubes with engineered pore structures and drug-delivery sites is possible with this scheme.

Emphasizing that he is designing "biological" materials (made from biological components) as opposed to "biomaterials" (made of synthetic polymers), $\mathrm{S}$. Zhang (MIT) presented a peptide scaffold for nerve-tissue engineering. The peptides self-assemble into a scaffold consisting of short peptide chains (8-24 units). These self-assembled peptides can be fabricated with any desired peptide sequence, forming dual-function chains with ionic bonds on one side and hydrophobic bonds on the other. This material has demonstrated effectiveness in neuron cell attachment, differentiation, and growth. Preliminary evidence of functional synapse formation between attached nerves was also reported.

R. Gilbert (CWRU) reported the development of 3D agarose gels to promote neurite growth. The scar formed when a nerve is cut has both physical and chemical impediments to growth. Specifically, chondroitin sulfate proteoglycan compounds tend to inhibit neurite extension. Conversely, laminin tends to promote growth. By mixing agarose with a crosslinking compound and then either laminin or chondroitin sulfate, the investigators were able to observe rates of nerve regeneration in vitro. They videorecorded the growth of E9 chick dorsal root ganglia through a microscope over a two-hour period. The ganglia cycled between growth, rest, and search modes. In the agarose-laminin gel, the neurites grew at a rate of $30 \mathrm{~mm} / \mathrm{h}$, while growth was much slower in the agarose-chondroitin sulfate gels, as expected.

\section{Nano-/Microstructured Materials}

In Symposium A, C.M. Lieber (Harvard) provided an overview of the fundamental properties and applications of SWNTs, including an application for analytical tools at the single-molecule level. Current microscopic probes are limited in resolution due to the diameter of the probe tip. By direct growth of SWNTs using metalcatalyzed CVD, subnanometer tip diameters may be achieved. On the device level, a suspended nanotube-crossed array that is bi-stable and addressable could be fashioned into a nonvolatile RAM device with speeds up to $200 \mathrm{GHz}$. Such units could form the basis for molecular-scale computers.

Using a vector model, E. Joselevich (Harvard) showed how electrical fields can be used to align nanotubes in a preferred direction. He identified two modes 


\section{Plenary Speaker de Gennes Touts Common Sense to Understand Soft Matter}

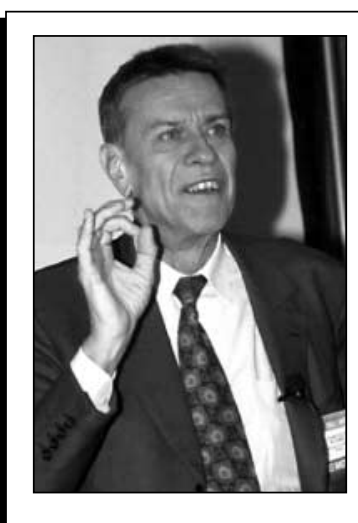

Nobel laureate Pierre-

Gilles de Gennes

(Collège de France) presented the Plenary address on soft matter at the 2000 MRS Fall Meeting in Boston.
The 2000 MRS Fall Meeting plenary presentation Monday evening was given by Nobel laureate Pierre-Gilles de Gennes of Collège de France. While acknowledging the theoretical basis for the behavior of materials in systems such as emulsions, block copolymers, and dewetting, de Gennes stressed the importance of common sense to reach a useful picture.

\section{Emulsions}

Emulsions form the structure by which oil and water can be held together. De Gennes showed two oil droplets separated by a thin sheet of water, with each droplet protected by a surfactant. If the droplets are held close together, theory says the surfactant molecules can be pushed out, so that the droplets come together and weld, no longer protected by the surfactant. "I think this is a myth," de Gennes said. While for poor surfactants this mechanism may happen, de Gennes argued that it is usually extrinsic effects that yield welding and coalescence. In real-life situations, he said, droplets nucleate because there is some dirt particle in the emulsion that serves as a nucleating agent. Dirt incorporation, indeed, is a common process of fundamental science, he said. A single germ added to a monodisperse emulsion can destroy the wall; this is followed by an "explosion." Then the germ "hops around." The motion of the germ is influenced by the capillary pressure, which transforms the initial sausage shape of the coalescing droplets toward a spherical shape. One then has one fat droplet growing in a sea of small droplets, and the germ runs on its outer surface. At later stages, as many droplets come together, a variety of things can happen. Accumulated water, due to lost surface, can stop the coalescence process, freezing in small and large droplets. Or the oil droplets can weld together between other droplets, forming small "bunches of grapes" floating in an oil slick. Much effort has gone into the "mythical process of intrinsic nucleation," de Gennes said, instead of considering realistic effects. There will always be dirt in an industrial reactor and in many laboratory experiments.

\section{Block Copolymers}

A block copolymer $A B$ is made of two distinct, successive chemical sequences, for instance, A: elastomer and B: polyamide.

In our days, there is a huge interest in block copolymers, but there is still room for simple inventions, said de Gennes. For instance, if we put our $\mathrm{AB}$ copolymer inside a polyamide matrix, we arrive at a soft core particle immersed in the nylon: The hope is that the mechanical properties of the nylon are imposed.

However, it often happens that the "brush" of B around the elastomer is too dense and does not interdigitate properly with the matrix: Then all is lost. A countermeasure (due to L. Leibler and co-workers) is to replace the $\mathrm{B}$ piece in the copolymer by a chemically different polymer $\mathrm{B}^{\prime}$. If we choose $\mathrm{B}^{\prime}$ so that it mixes spontaneously with the matrix, we get excellent particles.

\section{Wetting}

De Gennes spilled some water on a bare transparency (placed on his overhead projector). With enough water, he obtained puddles: The thickness of the puddles is well define, and results from a compromise between capillarity and weight.
Then de Gennes said that we should teach our students not only to observe nature, but also, immediately, to think about altering the conditions. He did this by squeezing the puddle with his fingers. The result is a film which is too thin, and which is not stable: Dry patches open up and grow. "With a good eye and a little faith," said de Gennes, "you will notice that the dry patches grow at constant speed."

The experiment corresponds to what is called dewetting. The fundamental laws for dewetting had been established some years ago by F. Brochard and co-workers.

Many extensions of this dewetting experiment are practically important. One of them is hydroplaning. The tires of a car on a wet road tend to be separated from the ground by a water film. But this film dewets: Dry patches grow between the asphalt and the tire. They must complete their task rapidly (in less than $15 \mathrm{~ms}$ ); if not, control is lost of the car.

This other form of dewetting has now been studied in detail. In a second stage, the situation where the car slows down (shearing the water film) has recently become understood.

Another example de Gennes gave related to plant protection, starting by examining a drop of water, carrying insecticide, hitting a leaf. When the droplet lands on the leaf, the water initially makes a flat drop, but then capillary forces cause it to spring back up, creating a kind of explosion and a secondary droplet. A suitable additive, for instance, a water-soluble polymer, causes the drop to spread, but not to fall back, so the secondary droplets are avoided. This reduces the amount of material wasted to the ground. Many airplanes that spray insecticides in the U.S. Midwest are now equipped with these magic solutions.

\section{Soft Matter: Looking Back}

Within soft matter, De Gennes observed that chemical innovation often comes before the physics. For instance, plastics have been made since the 1930s and were immediately recognized as very tough, mechanically. But it took about 60 years for researchers to understand in simple terms the source of this toughness. The field of adhesives is another example. Many innovations occurred between the 1920s and 1950, such as adhesives for high temperatures or for reducing atmospheres between metal surfaces. The inventions worked, but physicists are just coming to understand how adhesives work. Even now soft adhesives - tacky systems - are beginning to be understood, but hard adhesives, like epoxy glue, remain somewhat mysterious.

"Common sense is often more important than theory," de Gennes said. Dewetting experiments demonstrate this point. Describing this behavior using fluid mechanics can take years. Meanwhile, a few simple physical constructs can go far. In polymer science, he said, we have often suffered from too much knowledge. For instance, nuclear resonance spectra of polymers are so precise and detailed that the temptation has been to interpret them in all their detail, superimposing four or five different effects onto one spectrum, but possibly missing the simple picture in the process. He gave another example relating to wetting experiments in the offset-printing industry. This is an issue of printer, paper, and water. A very detailed theory of the complicated flow processes can be attempted, but it is the worker in the factory who must achieve the print quality. It is not the full hydrodynamic calculation that will help, but rather the use of simple scaling laws extracting one or two control parameters.

"So, theory can look powerful-and it can help bring insights-but it can go deeply astray (particularly when decorated by computers). We theorists should be very humble about our production," he said in conclusion. 


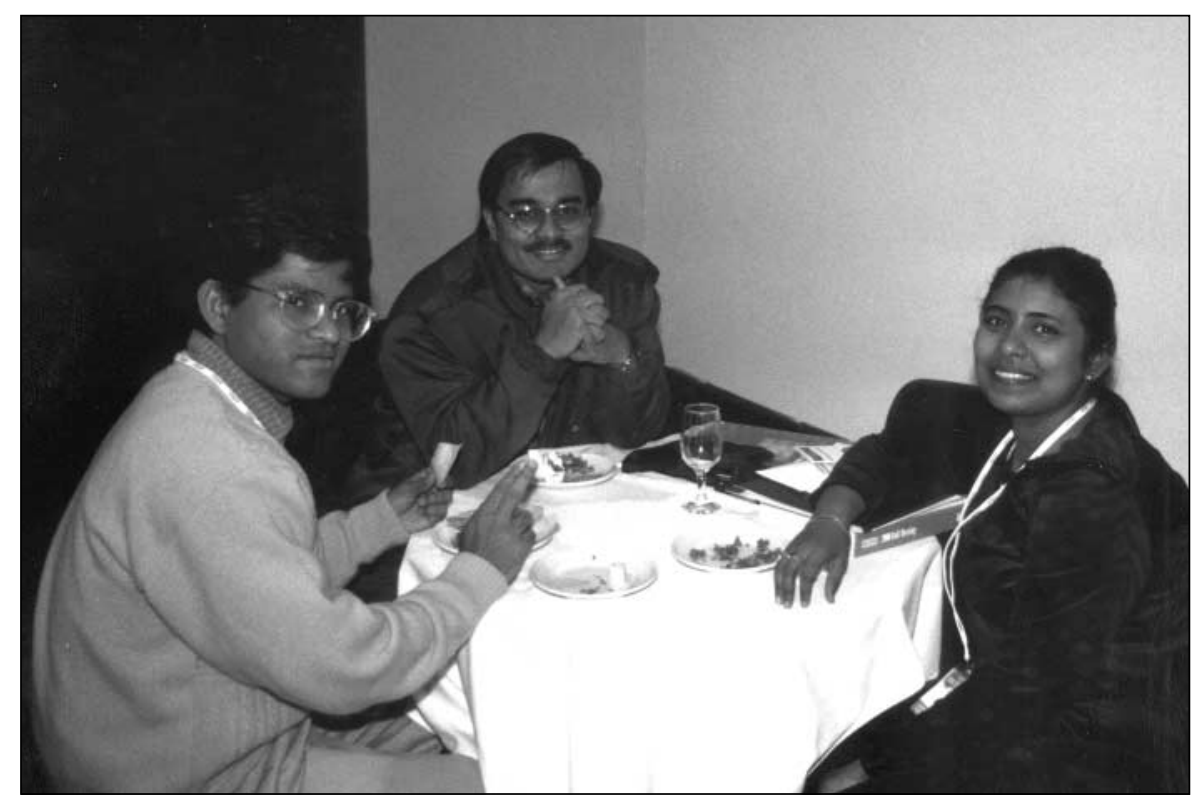

Students enjoy the opportunity to meet their peers during a Student Mixer held Sunday evening at the 2000 MRS Fall Meeting in Boston.

of growth of SWNTs on a substrate: surface growth, which produces wavy nanotubes that rarely align; and free growth, which yields straight nanotubes that align easily with the field. Using 8-nm ferritin monodispersed iron oxide nanoparticles adsorbed on $\mathrm{Al}_{2} \mathrm{O}_{3}$ as a patterning catalyst, application of an electric field between two surface-mounted electrodes caused the growth of aligned SWNTs of characteristic lengths greater than $15 \mathrm{~mm}$.

B. Wei (Rensselaer Polytechnic Institute) described an alternate means of producing aligned carbon nanotubes. His group used lithographic techniques to lay down a thin pattern of $\mathrm{Ni}$ on a silicon wafer substrate at $800^{\circ} \mathrm{C}$. CVD was performed using xylene as the carbon source. When the Ni patterns delaminated and lifted up from the surface of the substrate, aligned carbon nanotubes had formed between the bottom of the $\mathrm{Ni}$ films and the silicon substrate. This process offers the possibility for controlled growth of vertical, aligned nanotubes for practical devices.

Symposium B featured recent developments in nanolayered materials research. J.D. Embury (McMaster Univ.) emphasized the critical role of interfaces in controlling plasticity in nanostructured materials, drawing from key results in both vapor-deposited multilayers and codeformed composite wires. R.G. Hoagland and co-workers (LANL), using atomistic simulation, examined the strengthening effect of interfaces in metallic multilayers. The results reveal key insights on the character of the dislocation cores and the active participation of misfit dislocations in plasticity.

As demonstrated in Symposium C, unique properties of nanoscale materials are derived from the additional dimensional and compositional degrees of freedom that arise when the physical dimensions of the system are smaller than a characteristic length scale. One focus of the symposium encompassed the assembly of ordered 1D, 2D, and 3D arrays of anisotropic nanoparticles, a field that presents challenges not encountered with isotropic particles. Of particular interest were systems where specific chemical interactions (e.g., self-assembly) and electric fields (e.g., electrostatic trapping) were used to assemble ordered arrays from colloidal suspensions. B.D. Reiss (PSU), who received a Graduate Student Gold Award at the meeting, presented work that outlined the synthesis and directed assembly/manipulation of compositionally and geometrically anisotropic, noble-metal nanorods on planar and lithographically defined substrates. D.L. Feldheim (NCSU) discussed the importance of and techniques for addressing individual nanostructures (both electrically and optically) for technological applications such as nanoelectronics and biomedical engineering. Such measurements allow for the elucidation of the fundamental properties of nanomaterials such as electrical transport and tunneling.

In Symposium D, P.N. Prasad (SUNYBuffalo) described what can be done inside small places. For instance, inverted micelles, which are hydrophobic on the outside and hydrophilic on the inside, make prime sites for chemical reactions, increasing ion-pair interactions. Also, silica bubble "nanoclinics" holding optical or magnetic probes can be used for combining cancer diagnosis and targeted therapy. With the appropriate peptide hormones on the surface, the particles are programmed to go to the site of interest.

Symposium E demonstrated a clear shift away from III-V semiconductors toward silicon-based materials for microphotonics. This is motivated, on the one hand, by the move toward integrated optics applications that do not necessarily require emissive materials, and on the other hand, by the use of materials engineering to achieve more functionality from the silicon platform host. One of the major highlights of the symposium was the demonstration of coupled plasmon resonator waveguides, structures that guide and control light below the diffraction limit.

\section{Semiconductors}

Symposium G devoted a special session in honor of I. Akasaki (Meijo Univ.) and his achievements in the area of GaN. Akasaki's lecture on the renaissance of nitrides was introduced by recollections of K. Hiramatsu (Mie Univ.) on the chronological developments of the Akasaki group in Nagoya, Japan, and on the most recent advances in lateral epitaxy. Two more presentations by B. Monemar (Linkoping University) on the optical properties of quantum wells and by L.F. Eastman (Cornell University) on advanced microwave devices completed the scope of developments rooted in Akasaki's pioneering achievements. Dramatic enhancement of GaN crystalline quality by introduction of lowtemperature-deposited buffer layers and successful $p$-type doping were the results of Adasaki's perseverance in nitrides during the mid-1970s and the 1980s.

Symposium $\mathrm{H}$ focused on silicon carbide/silicon dioxide interfaces and their control, with several invited talks addressing different aspects of the problem. The most unexpected result was presented by researchers from $\mathrm{ABB}$ and the Linkoping University. H. Lendenmann (ABB) reported an increase of forward voltage drop in high-voltage $p-n \mathrm{SiC}$ diodes at current densities of $100 \mathrm{~A} / \mathrm{cm}^{2}$. The change was between 0.2 to $0.5 \mathrm{~V}$ and occurred over a period of several days. 
Concomitant with the voltage drop was the appearance of dark triangular features in the electroluminescence image, indicating a local decrease in the carrier lifetime. Complementary x-ray topography data presented by P. Bergman suggested that the change occurred within the SiC epilayer and is consistent with the formation of stacking faults. The defects were observed only in bipolar devices (not in Schottky diodes) and are likely due to energy released in recombination processes.

Work presented in the area of spintronics in Symposium I included the search for ferromagnetic semiconductors with high Curie temperature $T_{C}$, possibly well above room temperature. According to theoretical predictions, this is a feasible task. The synthesis of chalcopyrite material $(\mathrm{Cd}, \mathrm{Mn}) \mathrm{GeP}_{2}$ with $\mathrm{T}_{\mathrm{C}}$ above $300 \mathrm{~K}$ was reported by Medvedkin (Ioffe PhysicoTechnical Institute) and also by Joffe.

In Symposium J on quantum dots, the development of a mid-IR vertical cavity surface-emitting QD using the lead salt compounds PbSe and PbEuTe was reported. G. Springholz (Johannes Kepler Univ., Linz, Austria) described the device, which consists of two epitaxial high-reflectivity Bragg mirrors made of PbEuTe/ EuTe on either side of a 10-mm-thick selforganized PbSe QD superlattice. The PbSe islands are pyramidal in shape, with a base width of 250-400 $\AA$ and a height of 100-160 $\AA$, and show no electronic coupling. The high-refractive-index contrast of the mirrors gives the cavity a wide stop band. The central wavelength of the cavity is $4 \mathrm{~mm}$, and it exhibits a large number of modes with an energy spacing of $10 \mathrm{meV}$. Significantly, this device can operate up to $90 \mathrm{~K}$, making high-temperature QD IR lasers a possibility. R. Mirin (NIST) described the use of QDs for optical amplifiers. These SOAs have a typical gain of $30 \mathrm{~dB}$, suitable for power boosters for metro networks and LANs. QD technology was chosen because it offers relatively simple fabrication, large-gain bandwidth, and the ability to eliminate cross-gain modulation by uncoupling the QDs. These devices could compete favorably with gain-clamped SOAs.

\section{Device and Functional Materials}

The major emphasis of Symposium BB was the characterization and modeling of various types of microstructures such as twins, tweed, and antiphase boundaries in (mostly) martensitic and ferroelectric materials undergoing structural transformations. Researchers want to understand and then engineer these microstructures for optimizing desired functionalities. An interesting development was the study on the "domain-material properties relationship" in domain-engineered single-crystal PZN-PT and PMN-PT systems. By manipulating the domain structures, new symmetries can be created at the mesoscopic level, and the piezoelectric effect can be increased by almost an order of magnitude. Domain patterns also proved to be the key in the ferroelectric thin films that have lattice mismatch with the substrate. It was found that the two-dimensional cross-hatching domain patterns minimize the elastic energy of the whole system. Some evidence indicated that non $-180^{\circ}$ domain walls show some movement during switching, although the majority of the domain walls are pinned by the substrate in ferroelectric thin films.

In Symposium CC, K. Ito (NEC Laboratories) and A.I. Kingon (NCSU) discussed their work in which they used SPM-based piezoresponse imaging to study ferroelectric fatigue in PZT films. These results suggest that loss of polarization in individual $180^{\circ}$ ferroelectric domains is an important mechanism for fatigue in these films, in addition to (or perhaps instead of) domain wall pinning. R. Waser (IFF, Jülich) described an important advance in quantitative analysis of switching dynamics through electrical testing, whereby the contribution of time-dependent relaxation currents can be separated from the measured current resulting directly from domain switching.

The topics covered in Symposium DD included development of new materials, fabrication of thin films, the role of stress in thin films for understanding tunability, Curie temperature, loss tangent compared with bulk materials, and RF microstructural characterization techniques. D. Oates (Lincoln Laboratory) reported on progress of high-performance ferritetuned resonator (filter) devices at cryogenic temperatures that use high-criticaltemperature superconductors, and discussed the importance of using ferro/ paraelectric materials for trimming the device.

In symposium EE, A.H. Heuer (CWRU) discussed microstructure-property relations in MEMS materials. Using an on-chip micromachined Si microactuator capable of generating up to $700 \mu \mathrm{N}$ of force that they developed, Heuer's group measured bend strength, tensile strength, and fracture toughness at the wafer scale using electrostatic actuation. The researchers designed structures with zero residual stress and a zero residual-stress gradient and were able to make arbitrarily large structures that are extremely flat. They were also able to make predetermined shaped structures by controlling the residual-stress distribution, such as semicylindrical mirrors that can be flattened by electrostatic actuation.

T.W. Kenny (Stanford) gave a presentation on MEMS devices that can be used to measure a wide range of forces from micronewtons $(\mathrm{mN})$ to extremely small forces-attonewtons (aN, $\left.10^{-18} \mathrm{~N}\right)$-leading to new applications and interesting scientific measurements. Miniature commercial instruments, such as accelerometers, which measure very small forces (displacements) and pressure sensors, already operate near impressive levels: capacitive at $\mathrm{pN}$ in commercial parts; tunneling $\mathrm{pN}$ in custom (and soon commercial) parts; optical $\mathrm{pN}$ in commercial AFM; and aN in commercial parts. Materials research on the properties of $\mathrm{Si}$, a key MEMS material, is critical for optimizing and improving the performance of devices. MEMS device fabrication can support interesting science experiments as well as engineering system applications. J.P. Sullivan (SNL) presented an invited paper dealing with amorphous diamond as a new material for MEMS research. Amorphous diamond, or tetrahedral amorphous carbon, is a mixture of two phases: a fourfold-coordinated carbon phase mixed with a threefold-coordinated one. This new material, which is fabricated through pulsed laser deposition, typically consists of $70 \%$ of the fourfold phase and $30 \%$ of the threefold phase, although these proportions can be controlled to obtain desired properties. With an elastic modulus approaching $1 \mathrm{TPa}$, amorphous diamond materials could be instrumental in providing rubbing surfaces with long lives for MEMS applications.

Investigators at the Georgia Institute of Technology are using micromachining techniques to fabricate small-scale ceramic pressure sensors and combustion engines. M. Allen (Georgia Tech) described the development of a sensor to be placed at the outlet of the compressor in a turbine engine to monitor pressure changes. This extreme environment requires a device that can withstand temperatures up to $500^{\circ} \mathrm{C}$, pressures up to $50 \mathrm{bar}$, and pressure fluctuations occurring at a rate of $2 \mathrm{kHz}$.

\section{Materials Processing and Analysis}

In Symposium $\mathrm{P}$, topics ranged from growth and characterization of nanoscale surface structures to the growth and evolution of metallic, semiconducting, insulating, and organic thin films. In the session on surface magnetism, J. Kirschner (MPI Halle) described the creation of nanoscale magnetic dots, wires, and pillars including Co pillars on gold with a $\sim 3-\mathrm{nm}$ diameter and $\sim 6-n m$ height. H.C. Manoharan (IBM 


\section{Public Policy Boosts Materials Research}

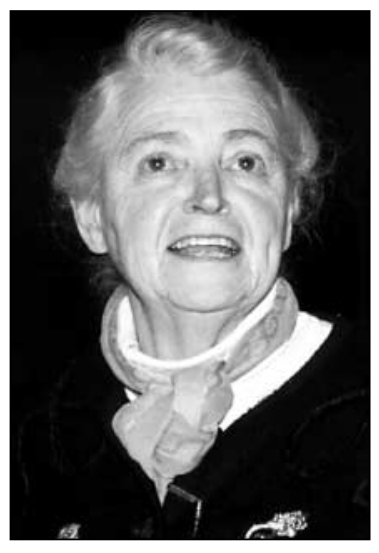

Mildred S. Dresselhaus, director of the $D O E$

Office of Science, gives a discourse on DOE's role in materials research.
A regular feature at MRS meetings are presentations from U.S. government funding agencies. Before the talks from the specific agencies, Merrilea J. Mayo (PSU) gave an overview of the status of federal funding for the mathematics, physical sciences, and engineering (MPSE) disciplines, and Mildred S. Dresselhaus (DOE) gave a discourse on DOE's role in materials research. Federal R\&D funding as a percentage of the U.S. gross domestic product has been in a long decline since the 1960s; the most recent sign of life was a spike in funding for the Strategic Defense Initiative (SDI) during the 1980s. While government spending for the life sciences is up $7 \%$ in recent years, spending for MPSE is down from $15 \%$ to $29 \%$, depending on the discipline.

Mayo said that this decline has major impact in three areas: the work force, future innovation, and economic growth. As funding declines, the quantity of students studying the physical sciences declines, which will lead to major shortages in the scientific work force in years to come. Such a lack of trained personnel will cause a drop in technological innovation, with a subsequent impact in economic growth.

To combat these trends, Mayo proposes a major increase in advocacy efforts from the MPSE community. "We are not good lobbyists," she said. Scientific organizations are widely dispersed, and they lack a coherent message. Furthermore, there are no grassroots organizations like the Sierra Club that brought the ecology movement to the forefront. Mayo calls for a single coalition led by the science and technology industries and encompassing such diverse groups as chambers of commerce, venture capitalists, and math and science teacher organizations. She said that this coalition should try to influence public opinion through op-ed pieces in local newspapers and articles in prominent national magazines. Focus groups should be assembled to target how the general public sees the science and technology community, she said, and polls should be taken to gather statistical data that could influence legislation. In general, the presence of a strong, attractive organization with a permanent staff dedicated to R\&D issues would go a long way toward solving the problem of declining federal funds. Citing both the opportunities and threats provided by a change in administration, Mayo said "the timing is perfect for action."

Using similar budget patterns, Dresselhaus, who is the director of the DOE Office of Science, expounded on the upturn of funding events in just the past two years. She reiterated that $\mathrm{DOE}$ is the major source of funding for materials research, along with NSF and DoD. In addition, DOE supports a number of R\&D facilities that are crucial for materials researchers. Her job of obtaining funds to place toward numerous initiatives has been easier with the significant backing of scientific societies such as MRS, university presidents, and others in the science community who have contacted Congress and senators. Dresselhaus expressed concern, however, over a recent finding that the top number of research articles published in Physical Review Letters and Physical Review-U.S.-based journals-are coming from Europe and the Far East. She commented that the United States, Europe, and the Far East are currently "among the leaders in materials research," and that it is important to remain within the leadership in certain research areas, or researchers will have a difficult time "catching up" and being effective in a new field. (See the February 2001 issue of MRS Bulletin, page 91, for Dresselhaus' article based on this presentation.)

To address funding opportunities in materials research in DOE, Iran Thomas, director of the Division of Materials Science, said his division is looking for innovative ideas within the funded programs. DOE national laboratories are restricted to submitting four proposals appropriate for the lab. Universities have no restrictions on the number of proposals submitted, and researchers may submit proposals for a single investigator. More specifically, the National Nanotechnology Initiative introduced within the past year cuts across three major government departments and agencies, including DOE. Of DOE's \$36 million increase, \$32 million consists of new money going into this initiative.

Tom Weber, director of the Division of Materials Research within the Directorate for Math and Physical Sciences at NSF, described new activities and grant opportunities. NSF is beginning to fund the U.S. portion of international materials research collaborations, predominantly with the European Commission. The Foundation has also established the Distinguished International Postdoctoral Research Fellowship program. Weber said that materials research is moving in the direction of international partnership, so NSF is concerned with preparing the next generation of students for international projects. He said that among the recommendations from numerous international workshops are developing virtual institutes through the Internet and video, developing exchange programs, creating a worldwide searchable database, establishing mechanisms for long-term collaborations, and enhancing public awareness of materials science and technology. He urged attendees to visit the NSF Web site for full grant information (www.nsf.org), and several directors were available to meet with attendees to discuss the grant process.

Craig S. Hartley, program director of Metallic Materials within Aerospace and Materials Sciences at the Air Force Office of Scientific Research (AFOSR) provided information about funding from AFOSR. He said that the Research Laboratory grants $19 \%$ of its funding to the Technology Directorates within AFOSR, $71 \%$ to universities, and $10 \%$ to industry. He advised potential principal investigators to check the Web site (www.afosr.af.mil) for Broad Agency Announcements that list grant opportunities, and to directly contact program managers. He said that AFOSR tends to support materials research that is linked to a specific device outcome or a specific materials problem.

The Advanced Technology Program (ATP) of NIST funds high-risk materials research. While government programs typically fund innovative ideas at the basic research level, and corporations tend to not pursue high-risk research, ATP helps accelerate the development of innovative technologies to the point that companies can invest their resources. These innovative ideas must show a broad national benefit. Michael Schen, program manager of the Electronics and Photonics Technology Office, and Donald Bansleben, program manager of Chemistry and Life Sciences, described a few new proposal initiatives at ATP. Since principal investigators can best decide when it makes good sense to pursue development of a particular technology, ATP accepts "rolling" proposal submissions, which means no deadline is set. ATP has also streamlined the proposal process and has initiated an electronic submission process. The ATP Web site can be found at www.nist.gov. 
Almaden) was able to create atomic mirages on a surface by placing an atom with a magnetic moment on a surface at one focal point of an elliptical corral formed by manipulating atoms with an STM. In a manifestation of the Kondo effect, a mirage of this atom was then formed at the other focal point with the same electronic properties.

Symposium Q emphasized the use of indentation techniques to solve materials problems and understand materials behavior. One of the highlights was a presentation by K.L. Johnson (Cambridge Univ.) who described the extensions of adhesive contact models to include viscoelastic deformation. Johnson's many contributions to contact mechanics include the codevelopment of the JKR theory for adhesive spherical contact. His latest efforts point the way for nanoindentation techniques to be extended beyond metals and ceramics to characterization of compliant polymeric and biological systems.

Microgravity, which is often associated with experiments in space, was brought "down to Earth" by N.I. Wakayama (Japan Science and Technology Corp.), in Symposium S, who reported on a method of growing protein crystals of adequate

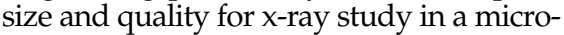
gravity environment on the ground. The method uses superconducting magnets to achieve a spatial distribution of magnetic strength to counteract the force of gravity. The work showed that it is possible to control vertical acceleration continuously from normal gravity to microgravity by applying an upward magnetization force on fluids. Bubbles in this environment behaved as if in a microgravity environment, expanding to fill more volume as the force decreased.

In order to noninvasively influence embryo development, J.M. Valles (Brown University) showed the effects of magnetic fields on the development of frog embryos. The magnetic field causes levitation by acting on the diamagnetic water molecules, thereby lifting all molecules in water. Likewise, a full frog embryo can be levitated in this way. Surprisingly, the result was not just an absence of effects due to gravity, but added effects were found due to the alignment of the magnetic field. Whereas cells typically divide alternately vertically and horizontally, in a magnetic field the embryo cells divided vertically; they aligned with the magnetic field like sections of an orange. Additionally, gastrulation (development of the gastrointestinal tract) is affected, causing greater damage as the time in a magnetic field is increased. N. Hirota (Univ. of Tokyo) described the achievement of lev- itation of nonmagnetic materials using an ordinary $10 \mathrm{~T}$ superconducting magnet with the aid of pressurized oxygen gas (magneto-Archimedes levitation). This process is a distinct way to separate glass particles of different densities in a liquid.

Symposium $\mathrm{T}$ covered a broad range of topics relating to structure and dynamics under geometric restrictions and introduced the dynamics in biologically oriented systems. Presentations were delivered on intercellular transport, transport in membranes, and biomolecules confined to nanopores. Researchers are also able to follow a single enzyme in time.

Symposium V introduced new types of electron microscopes to study materials problems that cannot be tackled by conventional electron microscopy. D.J. Stokes (Cambridge) demonstrated the potential of the ESEM to explore the behavior of complex fluids, including dynamic processes in emulsions and in situ formation of colloidal crystals. J.P. Craven (Cambridge) followed with some dramatic images of liquid drops wetting a variety of substrates. One of the key invited talks was given by D. Carson (Getty Conservation Institute) who highlighted the role played by ESEM in understanding the microstructural damage processes that lead to the degradation of ancient monuments such as the Mayan pyramids.

\section{Defects, Mechanics, and Length Scales}

Symposium $\mathrm{W}$ described computational methods to find the limits of strength, both at the elastic limit and via the behavior of interacting dislocations, and the measurement of limiting behavior at the extremes of small grain size (nanostructures) and small test volume (nanoindentation). Symposium $\mathrm{Z}$ highlighted advances in the theory and simulation of deformation and fracture at length scales from atomistic to mesoscale to continuum. E. Kaxiras (Harvard) described a quasicontinuum model with a constitutive relation derived by the density functional theory. He showed that it accurately predicts both mechanical and electrical response of a piezoelectric solid under indentation. S. Yip (MIT) presented a technique for coupling a classical molecular-dynamics simulation to an infinite linear elastic medium. Both techniques help make connections between atomic-scale mechanisms and the macroscopic mechanical response of materials.

Symposium AA overviewed various applications of computer modeling and/ or physical structural characterization for understanding oxide surfaces and interfaces. One example is the extrudation of

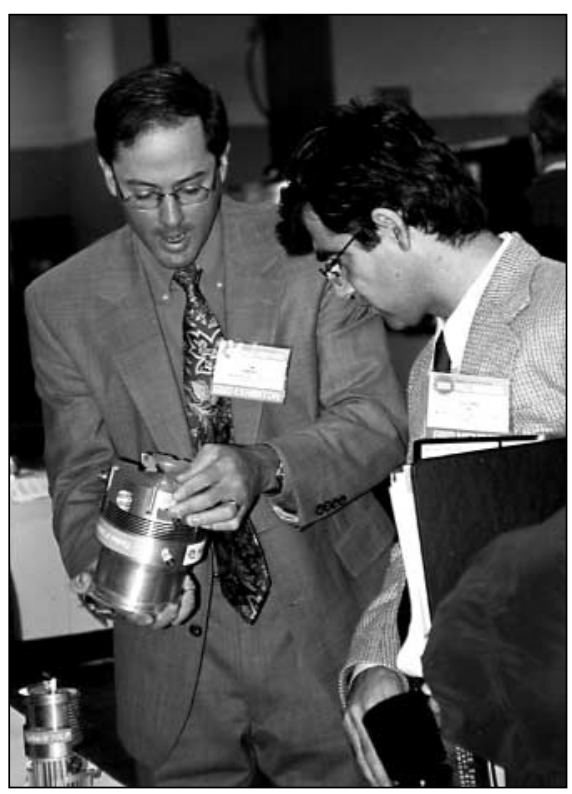

Meeting attendees visit the equipment exhibit that featured more than 225 exhibitors from around the world, displaying a range of equipment, services, products, publications, and software.

liquid aluminosilicate from polycrystalline alumina, presented by N. Ravishankar (Univ. of Minnesota). This study examined the possibility of removal of an intragranular glassy phase that was introduced to generate enhanced densification through liquid-phase sintering. It was shown by HRSEM that the extrudation depended on the wetting behavior of the free surfaces and the wetting behavior of the boundaries.

\section{Inorganic Materials}

Focusing on high-performance concrete, Symposium FF addressed a wide array of themes, including the microstructure, rheology, transport, shrinkage, and durability of concrete. The emphasis of Symposium GG was rational materials design, covering topics on novel synthetic methods leading to new materials, solving complex crystal structures using 4D crystallography, magnetic interactions in superconductors and colossal magnetoresistive solids, solid-state ionics (battery materials), microwave dielectrics, negative thermal expansion, and thermoelectric materials. Among the highlights, J. Tate (Oregon State Univ.) and A.W. Sleight (Oregon State Univ.) announced that their group had increased the conductivity for a $p$-type transparent conductor by a factor of 200 over that reported by other groups. Thin films of $\mathrm{CuCrO}_{2}: \mathrm{Mg}$ 
show a room-temperature conductivity as high as $285 \mathrm{~S} / \mathrm{cm}$ coupled with good transparency.

In Symposium HH, F.O. Witzel (Emitec Inc.) discussed innovations in automotive catalysts. While conventional automotive catalysts are based on ceramic honeycombs, his group based catalysts on thin metal ribbons, which have a lower thermal mass and thereby heat up more readily, leading to a reduction of start-up emissions. The metal ribbon is corrugated and wound into a cone. The group also introduced horizontal ridges on the ribbon in order to tailor the gas flow for optimum catalyst performance. M.J. Ledoux (Univ. of Strasbourg) described the synthesis of silicon carbide nanotubes, which, following impregnation with nickel nitrate, yield surprisingly active catalysts for the oxidation of hydrogen sulfide. Ledoux said that the activity may possibly be enhanced by capillary condensation in the nanotubes.

In Symposium II, G.A. Daniels (Univ. of Wisconsin) presented measurement of electromagnetic properties of undoped, Ca-doped YBCO, and oxygen-overdoped Ca-doped YBCO bicrystal films. The intergranular current density $\left(J_{b}\right)$ measured at $44 \mathrm{~K}$ for $5^{\circ}$ [001] tilt bicrystals showed improvements for the doped samples compared with undoped YBCO. The separation between the intragranular current density $\left(J_{\mathrm{c}}\right)$ and $J_{\mathrm{b}}$ almost disappeared for the oxygen-overdoped Ca-doped sample. The over-doped Ca-doped YBCO bicrystal behaved like a single crystal.

\section{Metals}

In Symposium K, researchers reported the discovery of a novel family of quasicrystals based on cadmium, adding about 10 binary and ternary phases to the already long list of quasicrystalline alloys. Whereas stable quasicrystalline phases were only known for ternary systems, $\mathrm{Cd}_{84} \mathrm{Yb}_{16}$ was reported to be thermodynamically stable. Possible applications of these materials include the use of quasicrystal-strengthened maraging steels for electric-razor shear heads and for surgical instruments and the use of quasicrystal powders to replace traditional fillers in polymer composites. Very low friction and wear coefficients have been measured.

In Symposium M, C. Leyens (DLRGerman Aerospace Center, Cologne, Germany) covered the R\&D status and needs for improved performance in electronbeam PVD thermal barrier coatings (TBCs). TBCs are currently used for lifetime improvement of highly loaded turbine blades and vanes in aeroengines and land-based gas turbines. Leyens described the importance of using a systems approach, considering both performance and durability and incorporating environmental and mechanical properties of each part of the TBC system and their interplay and changes during service. The challenge lies in maintaining good properties over a long time at high temperatures.

Titanium aluminides and refractorymetal silicides represent major classes of materials that were covered among others in Symposium N. In the area of magnetic effects, D. Wu (Dartmouth College) found strain-induced magnetism in FeAl single crystals and C.T. Liu (ORNL) reported that the softening of $\mathrm{Ni}_{60} \mathrm{Al}_{40}$ by iron additions was related to magnetic effects.

For further details about the technical content of the meeting, read the following symposium summaries and see the published proceedings.

\section{Synergy between Experiments and Simulations Emphasized in the Study of the Structure and Mechanical Behavior of Nanophase Materials (See MRS Proceedings Volume 634)}

The overall aim of Symposium B, Structure and Mechanical Properties of Nanophase Materials, was to bring together experimentalists working in this field and those carrying out computer simulations. Molecular-dynamics (MD) simulations now are able to treat mechanical behavior in samples with grain sizes up to $20 \mathrm{~nm}$, a size regime beginning to overlap the lower limits of many actual samples. MD simulations (A. Caro, Paul Scherrer Institute) show a distinct decrease in strength with decreasing grain size. C.C. Koch (NCSU) reviewed studies of the HallPetch behavior in nanocrystalline metals and noted several observations of such softening at the finest grain sizes that cannot be explained as experimental artifacts. MD simulations were carried out by P.M. Derlet and colleagues at PSI to predict the influence of two parallel free surfaces on the mechanical behavior of the thin foils used in TEM in situ straining experiments such as those described by A.K. Mukherjee (UC-Davis) and by R.C. Hugo, C.J. Youngdahl, and colleagues (LANL and Northwestern).

A number of talks captured exciting developments in nanolayered materials research. J.D. Embury (McMaster Univ.) emphasized the critical role of interfaces in controlling plasticity in nanostructured materials, drawing from key results in both vapor-deposited multilayers and $\mathrm{CO}^{-}$ deformed composite wires. R.G. Hoagland and co-workers (LANL), using atomistic simulation, examined the strengthening effect of interfaces in metallic multilayers.
The results reveal key insights on the character of the dislocation cores and the active participation of misfit dislocations in plasticity.

Enhanced superplastic behavior in alloys subjected to equal channel angular pressing was described by T.G. Langdon (USC), while H. Mughrabi (Univ. of Erlangen-Nuremberg) presented research into the fatigue properties of equal channel angular pressing material.

Symposium Support: JEOL, LANL, FEI Co., and ONR.

\section{Anisotropy Affects Behavior of Nanoscale Materials}

(See MRS Proceedings Volume 635)

The objective of Symposium C was to bring together the diverse scientific communities working on anisotropic nanoscale materials. It has been well established that the unique properties of nanoscale materials are derived from the additional dimensional and compositional degrees of freedom that arise when the physical dimensions of the system are smaller than a characteristic length scale of interest. Likewise, anisotropic particles can exhibit novel and enhanced properties compared with isotropic spherical particles. The symposium focused on a range of aspects of anisotropy on the nanoscale, including anisotropy resulting from shape (e.g., rods, nanowires, biomolecules), juxtaposition of different materials (e.g., nanoparticle heterodimers), compositional heterogeneity (e.g., core-shell particles, multilayer nanowires) and the intrinsic directionality of a probe (e.g., electromagnetism). Among the individuals driving this field are those involved in the assembly of value-added materials and devices from anisotropic nanoparticles. Chad Mirkin (Northwestern) detailed the unique aspects and advantages (e.g., speed, sensitivity, and selectivity) of employing nanoparticle assemblies for detecting DNA.

Another focus of the symposium encompassed the assembly of ordered 1D, 2D, and 3D arrays of anisotropic nanoparticles, a field presenting challenges not encountered with isotropic particles. Of particular interest were systems where specific chemical interactions (e.g., self-assembly) and electric fields (e.g., electrostatic trapping) were used to assemble ordered arrays from colloidal suspensions. B. Reiss (PSU), a recipient of this meeting's Graduate Student Gold Award, presented work that outlined the synthesis and directed assembly/manipulation of compositionally and geometrically anisotropic, noble-metal nanorods on planar and lithographically defined substrates. D. Feldheim (NCSU) discussed the importance of techniques 


\section{Boston High-School Students Explore Materials Research}

"Don't compete with the material because the material always wins." This is Bob Lange's message to those who are teaching concepts in materials science. The accuracy of this message was made evident by the group of high-school students brought to the 2000 MRS Fall Meeting to participate in a workshop sponsored by the MRS Outreach Subcommittee of the Public Affairs Committee. Students from three Boston high schools participated in these workshops, then toured the equipment exhibit area and the poster session at the MRS meeting.

Lange, of Brandeis University, introduced the students to the concept of materials science by explaining that the microscopic makeup of a material directs how the material behaves. Two "hands-on" activities were then led by Lange and Gay Kendall of Benét Laboratories, U.S. Army ARDEC.

In the first experiment, the students were instructed to design a lightweight material with the ability to push heavy objects. They received \#10 rubber bands, 3-in.long fettuccine noodles, and paper clips. The students were to produce a wand nearly $15 \mathrm{in}$. long that could be used to push a plastic container filled with water. Through this experiment, the students discussed how the structure of the material made it behave as it did-whether the wand bent or broke-and how reinforcement of the material made it behave differently.

The study on structure and behavior continued in another experiment, this time on polymers. The polymers workshop began with a discussion of three basic types of materials (metals, ceramics, polymers) whose differences were demonstrated with Styrofoam models to represent atomic structure. Students were then presented with a variety of common items made from polymer materials and asked to make observations about their physical properties at room temperature. Students appreciated the etymology of the word polymer when these properties were explained in terms of molecular chains. The students were asked to predict the behavior of polymers at very low temperatures. Heat was explained in terms of particle vibration, and liquid nitrogen was used to demonstrate the concept of glass transition. Students then made their own polymer materials from ingredients including white craft glue, borax, and cornstarch.

Members of the Outreach Subcommittee escorted the students through several exhibit booths to participate in demonstrations of instruments used in materials research. This tour was followed with another in the poster session. The students said that they enjoyed the workshop and interacting with researchers at the meeting. One student was considering going into the medical field and said that this workshop offered various possibilities in the research area. When the students were asked if the workshop has given them an idea of what a career in materials research would be
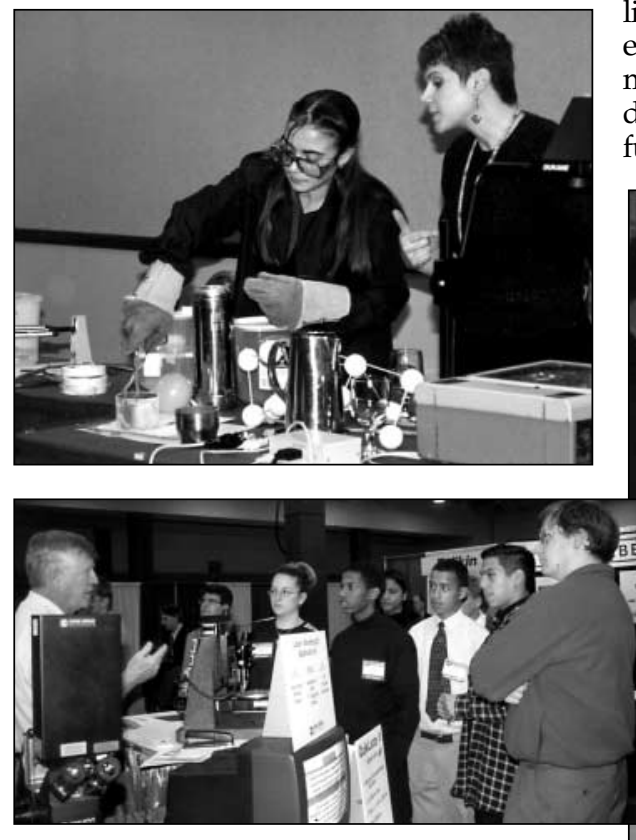

Boston high-school students explore materials research in a workshop sponsored by the MRS Outreach Subcommittee of the Public Affairs Committee. for addressing individual nanostructures (both electrically and optically) for technological applications such as nanoelectronics and biomedical engineering. Such measurements allow for the elucidation of fundamental properties of nanomaterials such as electrical transport and tunneling.

In this symposium, the theory, synthesis, processing, and property measurements associated with the chemistry of nanoscale organizations and assemblies were covered. The symposium mirrored cutting-edge research and the heightened awareness of nanotechnology (e.g., the applications of nanoparticle self-assembly in molecular electronics by T. Mayer's group at PSU). As the field matures, fundamental studies of nanoparticle properties will give way to the development of new applications and functional devices.

Symposium Support: Digital Instruments, Veeco Metrology Group; Surromed; NIST; ONR; and NSF.

\section{Nanofabrication Examined Broadly}

Encouraged by the successes of the 1999 MRS Fall Meeting symposia on nextgeneration lithographic processes for integrated circuits (ICs) with sub-100-nm resolution (Symp. J) and on non-lithographic approaches to micro- and nanofabrication (Symp. G), Symposium D on nanofabrication was organized with enough scope to create interactions between the two groups of researchers that participated in the 1999 symposia. Contributions from Lucent Technologies, IBM, Harvard University, NIST, and MPI (Halle and Stuttgart, Germany) showed the advantages inherent to self-assembly of colloidal particles, self-assembly of block-copolymers, and template-directed synthesis for nanostructure fabrication. Also, presentations from Harvard, Princeton, and Northwestern Universities suggested potentially important niche applications of soft lithography, nanoimprint lithography, and dip-pen nanolithography. For next-generation lithographies (NGL) that appear likely to be implemented by the traditional semiconductor industry, contributions from Lawrence Livermore National Laboratory, Lucent Technologies, and Fraunhofer Institut (Itzehoe, Germany) highlighted the necessity for developing exposure tools (extreme UV, electron and ion beams) and resists in parallel to produce ICs with sub-100-nm resolution. New advances in resists for NGL were emphasized by contributions from the Naval Research Laboratory and NTT (Japan), while nanocomposite resists jointly developed by the University of Connecticut and CERAMEC (France) appeared as a breakthrough. Molecular electronics was also 


\section{Materials Overview: MRS Awards and Symposium X Presentations}

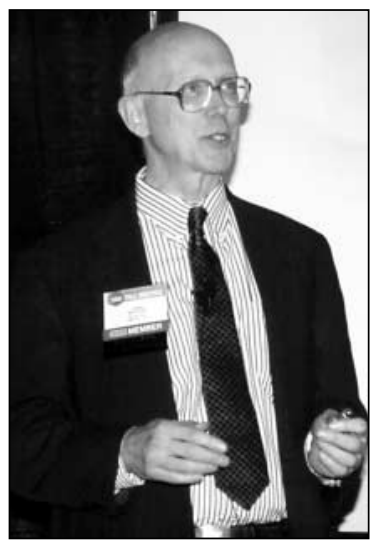

George M. Whitesides (Harvard) presents the Von Hippel address at the Awards Ceremony on Wednesday. He received the award for his research contributions on self-assembly.

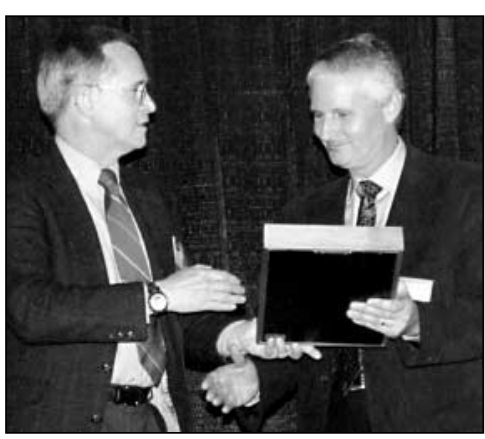

Anthony G. Evans (Princeton), right, receives the David Turnbull Lectureship, presented by MRS Immediate Past President Ron Gibala (Univ. of Michigan), awarded for bringing insights in mechanical behavior to materials engineering. compared with periodic structures, they are also less expensive to manufacture. Evans showed several examples of mechanicalproperty measurements. One of the features of these materials is that under compression along the axis of the material, there is no lateral expansion.

Samuel I. Stupp (Northwestern) focused his MRS Medal talk on self-assembly over multiple length scales to create functional materials. He described the assembly of molecules into supramolecular structures of less than $10 \mathrm{~nm}$. These can then self-organize to form films that integrate specific properties. He specified significant implications for biotechnology using this technique.

Dieter M. Gruen (ANL), in his MRS Medal talk, described the synthesis of ultrananocrystalline diamond films from $\mathrm{C}_{60}$ fullerene molecules (buckyballs). Traditional CVD diamond was made using methane and a large fraction of hydrogen. This forms large crystallites. However, recently it became possible to create large amounts of $\mathrm{C}_{60}$, which has had a significant effect for CVD diamond films. $\mathrm{C}_{60}$ fragments into a number of $\mathrm{C}_{2}$ dimers. These dimers can be deposited on a substrate to form nanocrystalline diamond, which has a microstructure made of randomly oriented 3-10-nm nanocrystals. These films have significant potential in tribology, corrosion resistance, and electrochemical electrodes, and they provide significant possibilities in MEMS applications. Furthermore, scientists have observed diamond nanocrystals in meteorites (carbonaceous chondrites).

In other overview talks, presented in Symposium X: Frontiers of Materials Research, Rena Bizios (RPI) described her work in biomaterials research as "proactive biomaterials" in contrast with previous work in which scientists used existing materialsdeveloped for other applications-as biomaterials. The current trend is to specifically create materials for bio-related applications. Bizios discussed the development of nanoceramics, including alumina, titania, and hydroxyapatite, and interactions with proteins.

Charles A. Vacanti (Univ. of

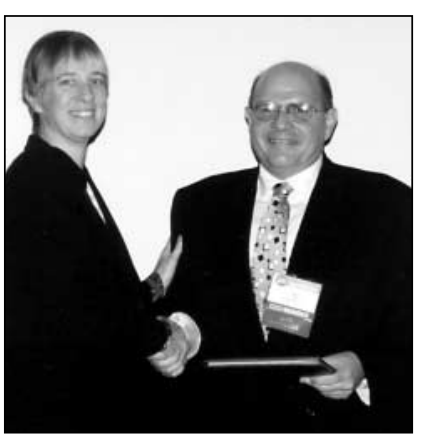

Samuel I. Stupp (Northwestern), right, receives the MRS Medal, presented by MRS Treasurer Merrilea J. Mayo (PSU), recognizing his work on supramolecular materials.

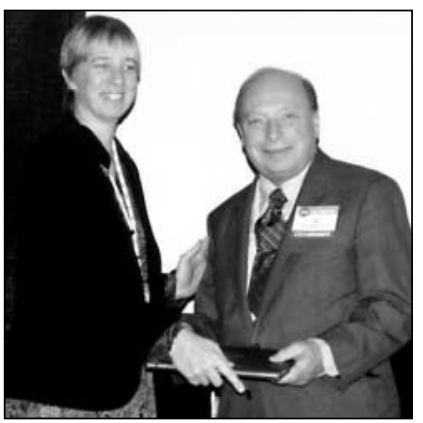

At the Awards Ceremony Wednesday night, Dieter $\mathbf{M}$. Gruen (ANL), right, receives the MRS Medal, presented by MRS Treasurer Merrilea J. Mayo (PSU). Gruen was recognized for his work on nanocrystalline diamond film. Massachusetts Medical Center), described his work dealing with the growth of cartilage using polymer scaffolding and chondracytes. When these systems are implanted in a living animal such as a rat, cartilage grows while the biodegradable scaffolding dissolves. The most famous example of this type of research is the human-ear-shaped scaffold implanted on the back of a mouse. Eventually cartilage was formed in the shape of the ear. Vacanti also described more recent success using liver cells, neurologic cells, and most recently, lung cells.

Describing criminal investigations, David Stoney (McCrone Research Institute) discussed the importance and limitations of forensic trace analysis, focusing primarily on the analysis of fibers. In another Symposium X talk, Randy C. Giles (Lucent Technologies) described the role of MEMS in future optical networks. He said that optical networks are in the process of replacing conventional copper-based networks primarily because of the higher bandwidth requirements due to the explosive growth of the World Wide Web. MEMS devices developed at his laboratory are based on flexure mechanisms. Giles described the use of micromachines in optical interconnects and optical switches. He showed arrays of mirrors that can be tilted to form the switches. 
well addressed by outstanding contributions from Harvard, Yale, and Rice Universities and UCLA as well, showing a fast-evolving field where nanofabrication techniques play a key role for actual device development. Photonic, electronic, and magnetic properties of nanostructures were reported: Key contributions from Lucent Technologies, IBM, MIT, Rice, and Princeton showed the industrial potential of micro- and nanodevices.

Symposium Support: IBM T.J. Watson Research Center; NEC Research Inst.; E-Ink Corp.; Lucent Technologies, Bell Labs; Duke Scientific Corp.; Nissei Sangyo America; Peacock Labs; Essilor Intl.; NSF; CERAMEC France; City Technology; ComSys GmbH; STMicroelectronics S.A.; and M. Braun.

\section{Microphotonics Shifts from III-V to Si-Based Semiconductors} (See MRS Proceedings Volume 637)

Presentations on microphotonics in Symposium E marked a clear shift in emphasis away from III-V semiconductors toward silicon-based materials. This is motivated, on the one hand, by the move toward integrated optics applications that do not necessarily require emissive materials, and on the other hand by the use of materials engineering to achieve more functionality from the silicon platform host. For example, cavity enhancement of $\mathrm{Si}$ :Er emission was demonstrated.

Overall, the fabrication of planar photonic crystals is now well established, and researchers are discussing refinements and showing impressive fabricated structures. One presentation described a characterization technique in which time-resolved measurements were used to identify the reflections inherently arising from the photonic crystal structure. Several speakers discussed enhancement of LED emission, and one group showed consistent improvement from electroluminescent microstructured devices. An important problem in guiding, which needs to be addressed more seriously (ie., scattering losses in photonic waveguides), was presented. Very strong extraction efficiency enhancements resulting from plasmon interaction were shown. A research group from Caltech demonstrated coupled plasmon resonator waveguides, structures that guide and control light below the diffraction limit.

Several talks were presented on photonic effects in self-assembled 3D crystals, that is, colloids and block copolymers. Challenges remain in achieving high dielectric contrast in the 3D photonic crystals and having large single crystals with low defect densities. The strong interaction between light and air spheres in a solid matrix was reported. Another presentation showed how colloid and emulsion templating could be used to make 3D photonic crystals. In contrast to most reports, in which high-volume-fraction colloidal assemblies are of prime interest, a presentation was given in which lowdensity charged colloids were stabilized by hydrogels. This approach produces crystals of higher quality, that is, lower defect density, and better long-range order at the expense of lower refractiveindex contrast than previously done. Possible applications are display devices and optical memories. Some novel approaches in replicating 3D colloidal assemblies in high-dielectric materials, including an approach of melt-imbibing chalcogenide glasses, were presented. The optical properties of three kinds of colloidal replicas, high-refractive semiconductors, metallo-dielectrics, and conjugated polymers were discussed. An alternative self-assembly strategy uses block copolymers and nanoparticles. This approach gives a high degree of freedom on photonic structure fabrication.

Symposium Support: Nanovation Technologies; Lucent Technologies, and Bell Labs.

\section{GaN Explored from Computational Fundamentals to Devices (See MRS Proceedings Volume 639)}

Symposium $\mathrm{G}$ on $\mathrm{GaN}$ and Related Alloys covered a wide span from computational fundamental physics over applied materials science to device design and system applications. Some of the highlights included the chemical analysis of gas-phase reactions during MOVPE, firstprinciples calculations of the most likely surface reconstructions, and experimental findings of surfactant and antisurfactant effects of $\mathrm{In}, \mathrm{Si}$, and $\mathrm{Mg}$, respectively. Their proper balance in combination with well-designed lateral epitaxial growth and low-temperature buffer and interlayer steps were shown to be crucial controls for superior crystalline quality. Advanced control of stress and strain also proved key to handling the huge polarization fields in the uniaxial system. In the range of several $\mathrm{MV} / \mathrm{cm}$, these fields significantly affect the electronic band structure, recombination dynamics, and carrier transport. Residual O donors and promising shallow Be acceptors were discussed in detail and an enhanced hole activation was demonstrated in the GaInN:Mg alloy over that of GaN:Mg. Record electron mobilities in MBE allowed the study of magnetotransport and the observation of the quantum Hall effect. Furthermore, luminescence across the entire visible spectrum was demonstrated by the size-tuning of
GaN/AlN quantum dots. Optimization of wave guiding in LEDs was shown to double light output, and advanced laser lift-off techniques have been applied to transfer laser diodes to $\mathrm{Cu}$ heat sinks, revealing benefits through thermal management. This progress was crowned by enhanced performances of light-emitting, detector, and electronic devices.

A special session was presented in honor of I. Akasaki (Meijo Univ.). Akasaki's lecture on the renaissance of nitrides was introduced by recollections of K. Hiramatsu (Mie University) on the chronological developments of the Akasaki group in Nagoya, Japan, and the most recent advances in lateral epitaxy.

Symposium Support: AIXTRON AG, Toyoda Gosei Co., Matsushita Electric Industrial Co., LumiLeds Lighting U.S., LLC, Uniroyal Optoelectronics, Xerox-PARC, Cree, and AFRL.

\section{SiC Growth and Processing Interfaces with Oxides and Defects}

(See MRS Proceedings Volume 640)

Symposium H reviewed recent developments in the field of silicon carbide growth and processing. The center of attention focused on silicon carbide/silicon dioxide interfaces and their control, with several invited talks addressing different aspects of the problem. Experimentally, the problem of low inversionlayer mobility on $4 \mathrm{H}-\mathrm{SiC}$ is linked to two main factors: (1) surface roughening arising from high-temperature implant annealing, and (2) a high density of interface states in the upper half of the bandgap on $4 \mathrm{H}-\mathrm{SiC}$. J. Williams (Auburn), working with L.C. Feldman and colleagues (Vanderbilt), has shown that postoxidation annealing in nitric oxide reduces the interface state density by as much as an order of magnitude. This is expected to lead to significant increases in electron inversion-layer mobility in SiC MOSFETs.

The most unexpected result was presented by researchers from $A B B$ and the Linköping University. H. Lendenmann reported the increase of a forward voltage drop in high voltage $p-n \mathrm{SiC}$ diodes at current densities of $100 \mathrm{~A} / \mathrm{cm}^{2}$. The change was between 0.2 to $0.5 \mathrm{~V}$ and occurred over a period of several days. Concomitant with the voltage drop was the appearance of dark triangular features in the electroluminescence image, indicating a local decrease in the carrier lifetime. Complementary x-ray topography data presented by P. Bergman suggested that the change occurred within the $\mathrm{SiC}$ epilayer and is consistent with the formation of stacking faults. The defects were observed only in bipolar devices (not in Schottky diodes) and are likely 
due to energy released in recombination processes.

Symposium Support: Cree, II-VI, MMR Tech., and Sterling Semiconductor.

\section{Spintronics Shows Promise (See MRS Proceedings Volume 641)}

Symposium I on semiconductor spintronics can be cast into four main topics. The first one concerns a search for ferromagnetic semiconductors with high Curie temperature $\mathrm{T}_{\mathrm{C}}$, possibly well above room temperature, which is a feasible task according to some theoretical predictions. Here, a synthesis of chalcopyrite material (Cd,Mn) $\mathrm{GeP}_{2}$ with $\mathrm{T}_{\mathrm{C}}$ above $300 \mathrm{~K}$ was reported (G.A. Medvedkin Ioffe, St. Petersburg). Other promising functional materials were shown to be hybrid structures of semiconductors and ferromagnetic metals, the latter in the form of overgrowth (e.g., half-metallic Heusler alloys, W. Van Roy, IMEC), inserted layers (e.g., MnAs, $\mathrm{H}$. Luo, SUNY-Buffalo; R.K. Kawakami, $\mathrm{UCSB}$ ), or MnSb inclusions. Remarkably, in such a granular system, magnetoresistance over $10,000 \%$ was discovered at room temperature $(\mathrm{H}$. Akinaga, JRCATNAIR, Tsukuba). The symposium presentations and discussions addressed the question of mechanisms controlling the steadily growing efficiency of spin injection into a semiconductor (D.K. Young, UCSB) as well as systems with long spin coherence time, suitable for quantum information devices. Here, GaAs/AlGaAs heterostructures grown along [110] (Y. Ohno, Tohoku Univ.) and Si/Ge heterostructures (F. Schäffler, Univ. Linz) are examples of promising approaches.

Symposium Support: ARO.

\section{List of Quasicrystal Phases Grows (See MRS Proceedings Volume 643)}

Quasicrystals continue to represent a challenge to solid-state physics, crystallography, and metallurgy, as evident in Symposium K. A novel family of quasicrystals based on cadmium has been discovered. This has added some 10 binary and ternary phases to the already long list of quasicrystalline alloys. One of these, $\mathrm{Cd}_{84} \mathrm{Yb}_{16}$, is thermodynamically stable. This is remarkable, since so far stable quasicrystalline phases were only known for ternary systems. Structure determination benefits from new phase-reconstruction techniques for $x$-ray single-quasicrystal data. Up to some 10,000 reflections can be measured and evaluated using high-quality samples of Al-based alloys obtained from Bridgman and solution growth techniques. The resulting structure models demonstrate that transition-metal-atom sites are sharply defined, while the Al- atom sites exhibit disorder, that is, the exact location of the atoms varies from place to place or the sites are left vacant. Concerning the surfaces, new He scattering data and atomic-resolution STM of AlPd-Mn quasicrystals are supporting the view that the quasicrystalline structure is maintained up to the surface where, however, the topmost layer is enriched in $\mathrm{Al}$ and depleted in Mn. Concerning applications, the use of quasicrystal-strengthened maraging steels for electric-razor shear heads and for surgical instruments is prominent. Very promising is the use of quasicrystal powders replacing traditional fillers in polymer composites. Remarkably low friction and wear coefficients have been measured. Quasicrystal research also has opened new prospects for the physics of classical alloys. The techniques developed in quasicrystal research are increasingly applied to investigate periodic metallic alloys with giant unit cells containing hundreds, and sometimes more than a thousand, atoms. For example, entirely new deformation mechanisms related to those found in quasicrystals have been discovered.

Symposium Support: ARO.

\section{Titanium Aluminides and Silicides High Among Intermetallics Receiving Attention}

(See MRS Proceedings Volume 646)

Symposium $\mathrm{N}$ contained a wide range of research ranging from $a b$ initio calculations to microstructural control and industrial applications. Almost a third of the presentations was devoted to titanium aluminides, which are starting to find applications in aircraft and automobiles. Refractory-metal silicides (with melting temperatures of the order of $2000^{\circ} \mathrm{C}$ ) were the second most heavily investigated material.

The titanium aluminides discussed consisted primarily of $\mathrm{TiAl}$ with $\mathrm{Ti}_{3} \mathrm{Al}$ as the secondary phase. The microstructures of these alloys can be widely varied and were shown to range from lamellar to equiaxed structures. Generally, the creep strength was reported to increase with decreasing lamellar spacing. W. Schillinger (Technical University of Hamburg-Harburg, Germany) showed, however, that a reduction of the average lamellar spacing from $200 \mathrm{~nm}$ to $25 \mathrm{~nm}$ was detrimental since such fine structures did not remain stable during creep. M. Takeyama (Tokyo Institute of Technology, Japan) used a combined experimental/ thermodynamic approach to determine phase diagrams in ternary Ti-Al-M (M = V, Nb, Cr, Mo) systems. Such phase diagrams are very important for controlling the microstruc- ture of such alloys.

Niobium and molybdenum silicides including disilicides (e.g., $\mathrm{MoSi}_{2}$ ) and 5-3 silicides (e.g., $\mathrm{Nb}_{5} \mathrm{Si}_{3}$ ) were discussed. In order to improve their fracture toughness, many of these silicides contained solidsolution Mo or $\mathrm{Nb}$ phases. J. H. Perepezko (Univ. of Wisconsin-Madison) described how to increase the silicon content in MoSi-B alloys (and thereby the oxidation resistance) without eliminating the solidsolution refractory phase. C.L. Fu (ORNL) presented $a b$ initio calculations demonstrating control of the thermal expansion anisotropy of $\mathrm{Mo}_{5} \mathrm{Si}_{3}$ alloys, resulting in significantly reduced microcracking.

Several magnetic effects were highlighted in this symposium. D. Wu (Dartmouth College) found strain-induced magnetism in FeAl single crystals. C.T. Liu (ORNL) found that the softening of $\mathrm{Ni}_{60} \mathrm{Al}_{40}$ by iron additions was related to magnetic effects.

Symposium Support: NASA Glenn Research Center; ORNL; and Chrysalis Technologies/PhillipMorris.

\section{Fundamental Physical Principles Applied to Nanostructures and Thin Films}

(See MRS Proceedings Volume 648)

Symposium $P$ focused on topics ranging from growth and characterization of nanoscale surface structures to the growth and evolution of metallic, semiconducting, insulating, and organic thin films. In all areas, a strong emphasis was placed on the understanding of fundamental physical principles.

Several of the presentations provided a stunning display of the current state of the art in nanoscale observation and manipulation. K. Takayanagi (Tokyo Institute of Technology) discussed quantized conductance of a gold nanowire pulled between a gold STM tip and a gold surface while showing TEM-generated movies of the atomic movement as the nanowire was thinned to a single-atom diameter. H.C. Manoharan (IBM-Almaden) was able to create atomic mirages on a surface by placing an atom with a magnetic moment on a surface at one focal point of an elliptical corral formed by manipulating atoms with an STM. In a manifestation of the Kondo effect, a mirage of this atom was then formed at the other focal point with the same electronic properties.

Surface magnetism was the focus on one session with many related presentations in other sessions. J. Kirschner (MPI-Halle) described results in the creation of nanoscale magnetic dots, wires, and pillars, including Co pillars on gold with a $\sim 3$-nm diameter and $\sim 6-\mathrm{nm}$ height. Other 
talks emphasized new approaches to characterization of magnetic thin films.

Nanostructure formation and properties were emphasized by many speakers. G.L. Kellogg (SNL) used LEED to show the evolution of $\mathrm{Pb}$ structures on $\mathrm{Cu}(111)$ from well-ordered islands to stripes. K.
Kern (MPI-Stuttgart) focused on selforganized growth of metal and semiconductor nanostructures including dots and wires in organized arrays. Thin-film epitaxy at a fundamental level was represented by talks such as that by $\mathrm{M}$. Ware (NCSU), who provided a detailed look at the growth of SiGe on Si substrates as a function of substrate orientation.

Symposium Support: SVT Associates, Thermionics Vacuum Products, Varian Associates, RHK Tech., k-Space Associates, ELMITECH Elektronenmikroskopie GmbH, Omicron Assoc., Surface Preparation Labs., ORNL, Epion Corp., and SPECS $\mathrm{GmbH}$.

\section{Poster Prizes Awarded at the $\mathbf{2 0 0 0}$ MRS Fall Meeting}

The 2000 MRS Fall Meeting Chairs awarded prizes for the best poster presentations. Prize recipients from poster sessions received $\$ 500$, a certificate, and the honor of having the winning poster displayed for the remainder of the meeting. Poster award recipients are (D9.32) Peptide Recognition and Nucleation of Semiconductor and Magnetic Materials, S.R. Whaley, C. Flynn, E. Gooch, J. Schaeffer, and A.M. Belcher (Texas-Austin); (D9.43) Fabrication of Photonic Crystals Using Metal Clusters as Nuclei for Self-Formation of Etching Masks, T. Tada, T. Kanayama, and V.V. Poborchii (JRCAT); (F5.33) Nanocrystalline Si Void-Column Network Thin Films Deposited by High-Density Plasma: Application to High Sensitivity and Ultrafast Humidity Monitoring, A.K. Kalkan, H. Li, and S.J. Fonash (PSU); (G6.13) IR-VUV Dielectric Function of $\mathbf{A l}_{1-\mathbf{x}} \mathbf{I n}_{\mathbf{x}} \mathbf{N}$ Determined by Spectroscopic Ellipsometry, A. Kasic and M. Schubert (Univ. of Leipzig and Nebraska Univ.), B. Rheinländer (Univ. Leipzig), J. Off and F. Scholz (Univ. of Stuttgart), and C.M. Herzinger (J.A. Woollam Co., Lincoln, Nebraska); (I.32) New Magnetic-Nonmagnetic Semiconductor Heterostructure $\mathrm{Cd}_{1-x} \mathbf{M n}_{\mathbf{x}} \mathrm{GeP}_{2}-\mathrm{CdGeP}_{2}, \mathrm{G} . \mathrm{A}$. Medvedkin (Ioffe PhysicoTechnical Inst.) and T. Ishibashi, T. Nishi, and K. Sato (Tokyo Univ.); (N5.23) Deformation Twins in $\mathbf{N i}_{3} \mathbf{N b}$ Single Crystals with DO a $_{\text {a }}$ Structure, K. Hagihara, T. Nakano, and Y. Umakoshi (Osaka Univ.); (P3.13) Spiral Growth in Molecular-Beam Epitaxy Far from Thermodynamic Equilibrium: Scaling Behavior and Growth Mode Transitions, K. Wiesauer and G. Springholz (Johannes Kepler Univ., Linz, Austria); (P11.36) Smoothening of (001) and (111) Cu Films Epitaxially Grown on Si Substrates, R.A. Lukaszew, C. Uher, and R. Clarke (Univ. of Michigan); (W5.11) Plastic Deformation at Micron Scale in Al and Cu Thin Films Studied by Microbeam X-Ray Diffraction (mXRD), N. Tamura, A.A. MacDowell, R.S. Celestre, H.A. Padmore, and B.W. Batterman (LBNL), J.R. Patel (LBNL and Stanford), R. Spolenak, M.D. Morris, and W.L. Brown (Bell Labs/Lucent), B.C. Valek and J.C. Bravman (Stanford), and T. Marieb (Intel); (Y8.3) Evolution of Microstructure and Defect Structure during Nucleation and Growth of the L10 Phase in Manganese-Aluminum, C. Yanar, J.MK. Wiezorek, and W.A. Soffa (Univ. of Pittsburgh) and V. Radmilovic (Univ. of Belgrade); (CC5.3) Direct Comparison of Structural and Electrical Properties of Epitaxial (001)- , (116)- , and (103)Oriented $\mathrm{SrBi}_{2} \mathrm{Ta}_{2} \mathrm{O}_{9}$ Thin Films, H.N. Lee, A. Visinoiu, S. Senz, N.D. Zakharov, A. Pignolet, and D. Hesse (MPIMikrostrukturphysik-Halle/Saale); (CC10.1) Structural Distortion and Ferroelectric Properties of $\mathrm{SrBi}_{2}\left(\mathrm{Ta}_{1-x} \mathrm{Nb}_{x}\right)_{2} \mathrm{O}_{9}$, Y. Shimakawa and Y. Kubo (NEC) and Y. Tauchi, T. Kamiyama, and H. Asano (Univ. of Tsukuba, Japan); (GG3.25) Interference Effect Between Electron and Ion Flows in Semiconducting $\mathrm{Fe}_{3} \mathrm{O}_{4}$, H.-I. Yoo and J.-O. Hong (Seoul National Univ., Korea); (GG9.28) Electrodeposition of Thermoelectric Nanowire Composites, A.L. Prietol, M. Martín-González, J.F. Behnke, M.S. Sander, A.M. Stacy, R. Gronsky, and T. Sands (UC-Berkeley);
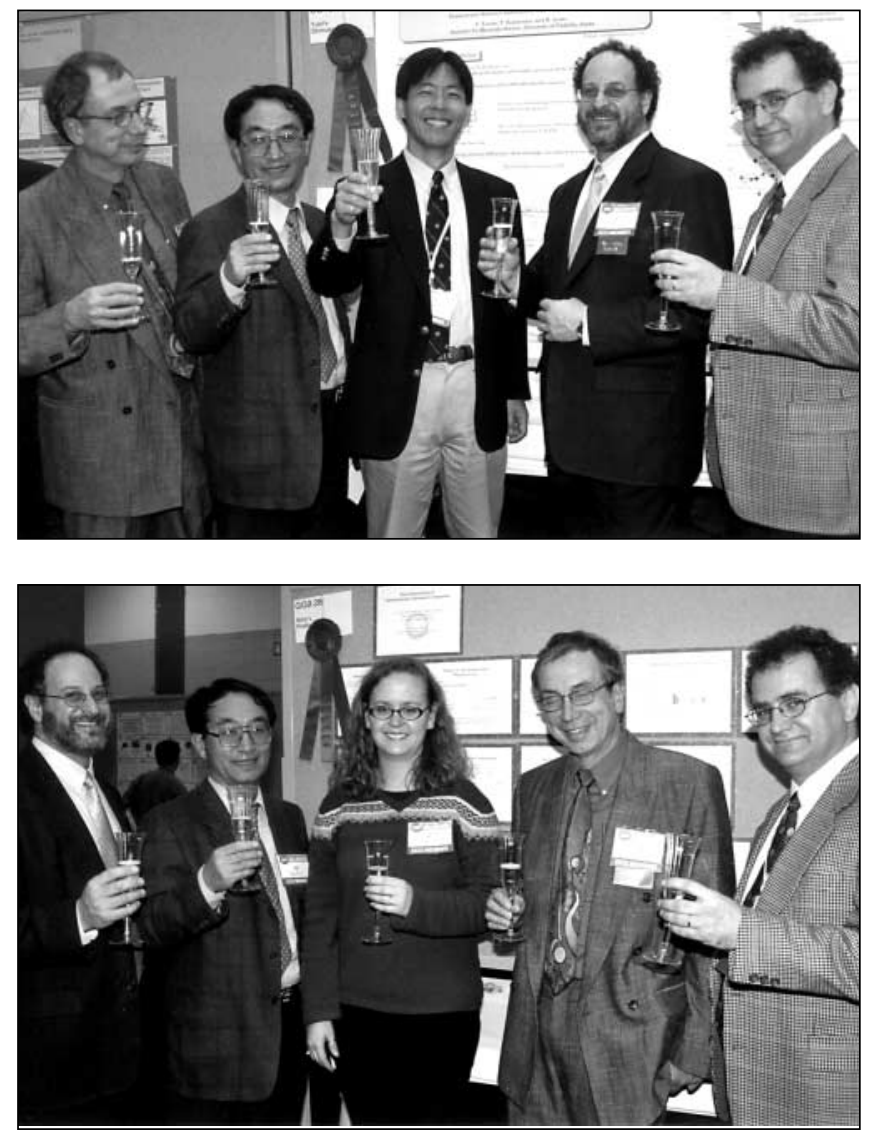

Poster Prizes are awarded by the Meeting Chairs at the 2000 MRS Fall Meeting in Boston.

(HH4.12) A Novel Catalyst Preparation for Dechlorination of Chlorinated Hydrocarbons, Y.S. Cho, J.C. Park, B.H. Lee, and J. Yi (Seoul National Univ., Korea); (II9.18) High-Temperature Superconducting Tapes Deposited by the Non-Vacuum, LowCost Combustion Chemical Vapor Deposition Technique, M.K. White, S.L. Krebs, Y. Xue, A.C. King, I.H. Campbell, T.A. Polley, D.S. Mattox, and S.S. Shoup (MicroCoating Technologies, Chamblee, Georgia); (JJ5.19) New Transparent Substrate with Silica Aerogel Layer for Surface-Emissive Devices, $\mathrm{H}$. Yokogawa, K. Kawano, and M. Yokoyama (Matsushita Electric Works, Osaka, Japan), T. Tsutsui and M. Yahiro (Kyushu Univ., Fukuoka, Japan), and Y. Shigesato (Aoyama Gakuin Univ., Tokyo, Japan); and (LL5.5) Photocrosslinked Poly(Propylene Fumarate) Scaffolds for Orthopedic Applications, J.P. Fisher, T.A. Holland, and A.G. Mikos (Rice Univ.). 


\section{Nanoindentation Gains Maturity as Technique for Studying Mechanical Behavior}

(See MRS Proceedings Volume 649)

Symposium Q highlighted the maturation of the field of nanoindentation and nanotribology, as considerably less emphasis was placed on techniques and analyses for depth-sensing indentation and more emphasis was placed on the use of indentation techniques to solve materials problems and understand materials behavior. Although technique development continues to be central in this field, accepted procedures for instrument and probe calibration and deconvolution of nanometer-scale load-displacement data in terms of underlying materials properties are now in wide use. Presentations were made demonstrating extensions of nanoindentation methods to include viscoelastic contacts, hard coatings on soft substrates, 3D finite element models, dissipated-energy-based analyses, the use of loading and unloading slopes, measurement of residual stresses, and direct comparison of indentation-derived properties with those from bulk tests.

Y.T. Cheng (GM) used finite element models to discuss general energy-based methods for the analysis of loaddisplacement traces obtained with sharp indenters. Although a universal relationship appears to exist connecting energy recovered during an indentation contact to the materials modulus/hardness ratio, the unique deconvolution of loaddisplacement data to obtain a materials stress-strain curve in the presence of work hardening is not possible. T.F. Page (Newcastle) and G.M. Pharr (Univ. of Tennessee and ORNL) presented results demonstrating the ability of spherical indenters to obtain stress-strain responses of bulk and thin-film materials, including characterization of residual stresses in metals and hard films on soft substrates. Indentation size effects (the apparent variations in hardness with contact load) and their interpretation in terms of yieldstress variations with the scale of the deformation or with plastic strain gradients still continue to be controversial. W.D. Nix (Stanford) discussed variations in the hardness of Mo, Ta, and $\mathrm{Au}$ in terms of strain-gradient plasticity and the theoretical yield stress for these metals. Estimates of the theoretical yield stress for Ir, based on a spherical indentation method, were presented by J.G. Swadener (Univ. of Tennessee and ORNL). The use of focused ion-beam techniques to section nanometer-scale indentations appears to hold great promise for both scanning and transmission microscope specimen preparation. J. Bradby (Australian Natl. Univ.) presented results describing the dislocation and phase-transformationmediated plasticity that occurs during indentation of $\mathrm{Si}$, combining nanoindentation and Raman spectroscopy with focused ion-beam-prepared TEM sections

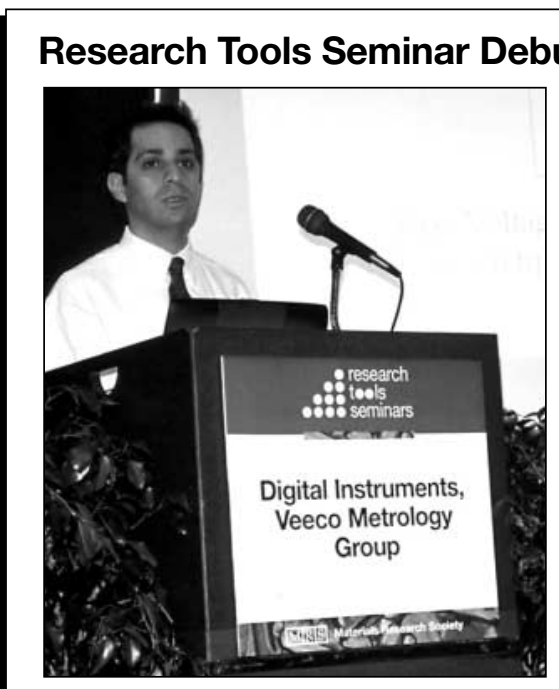

A representative from Digital Instruments, Veeco Metrology Group presented a Research Tools Seminar on advanced tools for nanoscale measurement of electrical properties of materials.

to arrive at a complete picture of the deformation of Si. A highlight of the meeting was a presentation by K.L. Johnson (Cambridge) describing the extensions of adhesive contact models to include viscoelastic deformation. Johnson's contributions to contact mechanics are many and include the codevelopment of the JKR theory for adhesive spherical contact and the publication of the book Contact Mechanics. His latest efforts point the way for nanoindentation techniques to be extended beyond metals and ceramics to the characterization of compliant polymeric and biological systems.

Symposium Support: Hysitron, Micro Photonics, MTS Systems Corp., Nano Instruments Innovation Center, Applied Materials, and Hysitron.

\section{Magnetic Field Effects Range from Levitation to Alignment}

Symposium $S$ attracted researchers working on the processing of not only magnetic but also nonmagnetic materials, including diamagnetic and paramagnetic materials, in an attempt to expand the boundary of materials processing using applied magnetic field. One of the main topics was diamagnetic levitation. Beaugnon (CNRS-CRETA) demonstrated that the Moses effect (change of the liquid surface under a magnetic field gradient) was enhanced by combining it with an applied flow, which was successfully explained theoretically. Creation of a strong field gradient is of primary importance for the application of levitation to materials processing and separation. A simple but powerful method was presented by K. Shinohara (JSTC, Japan). According to Shinohara's group, the insertion of a ring of steel into a moderate magnetic field can generate a surprisingly strong field gradient, for example ca. $1400 \mathrm{~T}^{2} / \mathrm{m}$, necessary for the levitation of a water droplet. The smaller the inner diameter of the ring, the stronger becomes the gradient. J.M. Valles (Brown Univ.) reported the effect of microgravity generated by levitation on frog embryos. In addition to the gravitational effect, they showed that the magnetic field also affects the internal structure of the embryo through magnetic orientation.

Texture formation and alignment of metals, ceramics, and polymers, for example, under a magnetic field was another focus of interest. Nanotube alignment was reported by Y. Tanimoto (Hiroshima Univ.). Interestingly, the same topic was presented in Symposium A, suggesting the relevance of applied magnetic fields for the control of nanoparticles.

Symposium Support: ARO. 


\section{Small Spaces Form Unique Places for Materials Phenomena}

(See MRS Proceedings Volume 651)

Symposium $\mathrm{T}$ covered a broad range of topics relating to structure and dynamics under geometric restrictions. Emphasis was on methods of probing confined systems, diffusion in porous media, polymers and membranes, dielectric and mechanical relaxation in nanopores, rheology and friction studies of embedded liquids, and properties of dendrimer supermolecules. Speakers shared different points of view on the fundamental questions of how spatial restrictions modify a system to behave significantly different than in the bulk, how this difference relates to the molecular properties, and how it can be probed. There appears to be a continuing interest in the dynamics and thermodynamics of confined molecular systems.

The aspect of the dynamics in biologically oriented systems was introduced. Presentations were given on intercellular transport, transport in membranes, biomolecules confined to nanopores, and following a single enzyme in time.

Of special interest was the session on nanorheology and friction, where experiments and theoretical approaches were discussed. The possibility was raised that sheared liquids between nearby surfaces may, in the sliding state, display processes on many scales. The underlying mechanism for this hierarchical behavior is still not known. New analytical and numerical (molecular dynamics) results have been introduced, proposing frameworks for understanding structure of liquids at interfaces, static friction, shear thinning, mechanisms of energy dissipation, and phase transitions under confinement. Methods to control friction have been discussed that enable researchers to eliminate chaotic stick-slip motion and modify frictional forces. Clearly, despite new experimental and theoretical investigations, a microscopic-level understanding is still missing. The need for new approaches has been recognized, and the possibility of introducing optical probes as sensor of local dynamics was discussed.

Other sessions dealt with various ways to probe the nature of liquids near interfaces. Notable among these methods were $x$-ray reflectivity and grazing incidence diffraction experiments, AFM, surface force apparatus, NMR, and dielectric relaxation. Glass-forming fluids have proven interesting in the investigations of the role of confinement on ordering and phase transitions.

Recent advances in dynamics of wetting were covered. Spreading of ultrathin liquid films on solid surfaces and the influence of wetting properties on diffusion in confined systems have been discussed. Direct experimental studies have found a relation between slip effects and wetting at fluid-solid interfaces.

Symposium Support: MMR Tech., ONR, Exxon, and Tel Aviv Univ.

\section{Low Vacuum SEM Used to Study Wet, Ancient, or Insulating Materials}

Symposium V on low-vacuum SEM/ ESEM in materials science focused on using this new breed of electron microscope to study materials problems that cannot be tackled by conventional electron microscopy. D.J. Stokes (Cambridge) demonstrated the potential of the ESEM to explore the behavior of complex fluids, including dynamic processes in emulsions and in situ formation of colloidal crystals. J.P. Craven (Cambridge) followed with some dramatic images of liquid drops wetting a variety of substrates. Moving to oily specimens, two presentations on the characterization of asphaltenes were given by E.T. Romero-Guzman and G.A. Camacho-Bragado (Instituto Nacional de Investigaciones Nucleares, Mexico). One of the key invited talks was given by D. Carson (Getty Conservation Institute), who highlighted the role played by ESEM in understanding the microstructural damage processes that lead to the degradation of ancient monuments such as the Mayan pyramids.

Another presentation featured work that exploits the ability of these instruments to examine uncoated insulators and poorly conducting specimens. In particular, a segment of one session was dedicated to applications in several areas of electronic materials, including electronic polymer devices (D.S. Thomas, Cambridge), GaN (M. Toth, Univ. of Technology, Sydney), silicon devices (M. Phillips, Univ. of Technology, Sydney), metrology (D.C. Joy, Univ. of Tennessee), and insulating materials (B.J. Griffin, Univ. of Western Australia).

Symposium Support: JEOL and FEI Co.

\section{Limits of Strength Approached from Theoretical and Experimental Perspectives}

Symposium $W$ considered both the upper and lower limits of strength in real materials and included both theoretical and experimental approaches to the problem. The "limits of strength" has been an active subject of research and speculation for the better part of a century. The problem has recently assumed new importance, for two reasons. First, given recent advances in ab initio techniques and computing machines, the limits of strength can (sometimes) be calculated with considerable accuracy, making this one of the very few problems in mechanical behavior that can actually be solved. Second, with recent advances in materials engineering, the limits of strength are being approached in some systems, such as hardened or defect-free films, and their relevance is becoming recognized in others, such as hard coatings, carbonitrides and diamond-cubic crystals, ultrafinegrained structural metals and alloys, and devices that operate at the smallest sizes or at the margins of reliable performance. This symposium assessed the current understanding of the limits of strength and the nature of failure, their prediction and control in practice, and their exploitation in the design of advanced engineering devices. Among the technical highlights were the descriptions of new computational methods to find the limits of strength, both at the elastic limit and through the behavior of interacting dislocations, and the measurement of limiting behavior at the extremes of small grain size (nanostructures) and small test volume (nanoindentation).

\section{Atomic-Scale Mechanisms and Macroscopic Mechanical Response Linked through Multiscale Modeling (See MRS Proceedings Volume 653)}

Symposium Z highlighted recent advances in theory and simulation of deformation and fracture at length scales from atomistic to mesoscale to continuum. Many talks were devoted to plastic deformation and work hardening, particularly the mystery of size effects in plasticity. E. Van der Giessen (Delft Univ. of Technology) reported that a discrete dislocation model in 2D shows size effects in shear deformation, and he compared his results with the predictions of strain gradient theory. M. Baskes (LANL) presented 2D molecular-dynamics simulations of shear deformation, also showing size effects, in this case arising from surface nucleation of dislocations.

D. Hughes (SNL-Livermore) presented TEM micrographs of dislocation patterns in fcc metals and showed data on universal scaling behavior in both the spacing and misorientation distributions associated with different types of boundaries. E. Holm (SNL-Albuquerque) described how the competition between enthalpy and entropy governs the evolution of boundaries and gives rise to this scaling behavior. She noted that the same scaling behavior is observed in many contexts, including the spacing of galaxies.

Two particularly striking innovations in multiscale modeling techniques were 


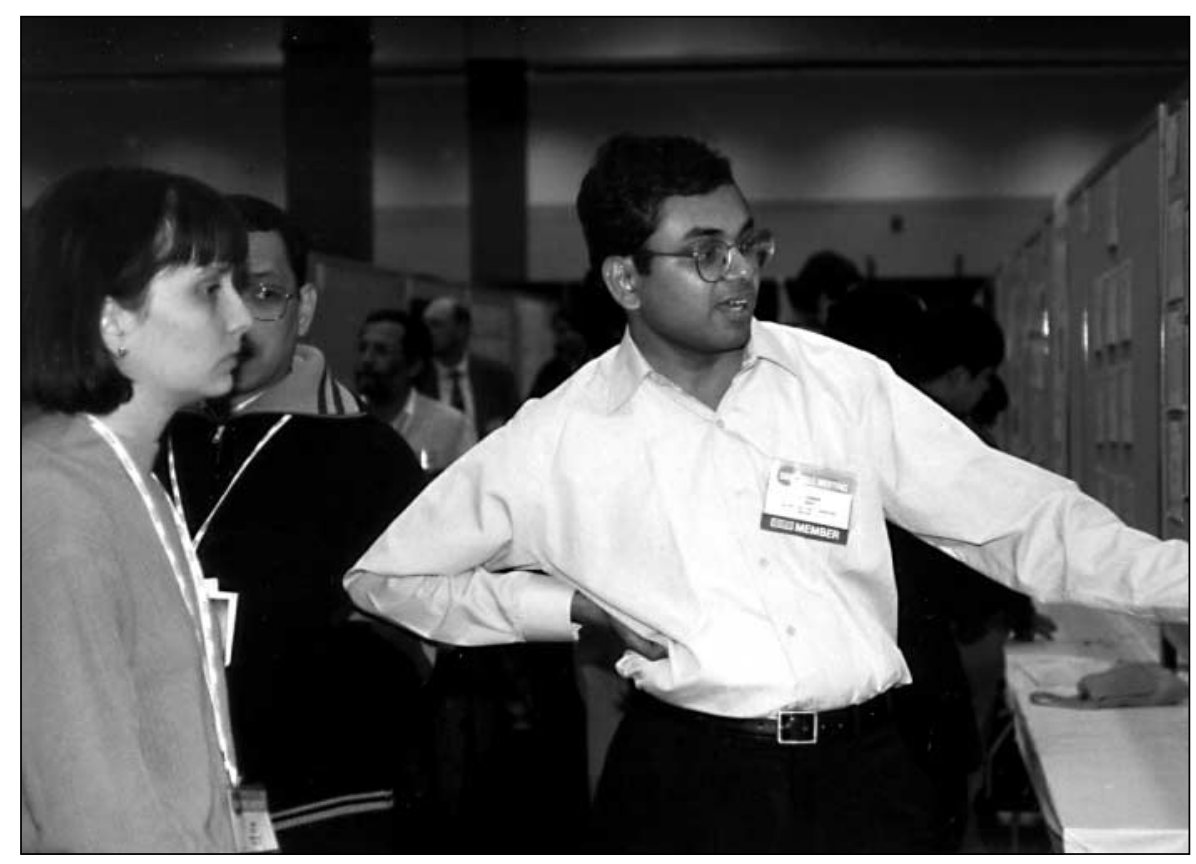

Meeting attendees discuss their research during the poster sessions at the 2000 MRS Fall Meeting in Boston.

presented. T. Kaxiras (Harvard) described a quasi-continuum model with a constitutive relation derived through density functional theory and showed that it accurately predicts both mechanical and electrical response of a piezoelectric solid under indentation. S. Yip (MIT) presented a novel technique for coupling a classical molecular-dynamics simulation to an infinite linear elastic medium. Both techniques help make connections between atomic-scale mechanisms and macroscopic mechanical response.

Symposium Support: ARO, NIST, and ONR.

\section{Oxide Surfaces and Interfaces Studied (See MRS Proceedings Volume 654)}

Symposium AA provided overviews of various applications of computer modeling and/or physical structural characterization to understanding oxide surfaces and interfaces. U. Diebold (Tulane Univ.) contrasted the results of a STM study of several oxide surfaces with first-principles, total-energy calculations of these surface structures. D. Bonnell (Univ. of Pennsylvania) discussed the nature of $\mathrm{SrTiO}_{3}$ bicrystal grain-boundary-surface junctions as revealed by the potential distribution measured by electrostatic force microscopy and scanning surface potential microscopy. This study yielded information on the atomic structure of the grain boundary and the segregation of dopants as well as the transport properties along these grain boundaries.

The versatile Perovskite system $\mathrm{SrTiO}_{3}$ was discussed by R.F. Klie (Univ. of Illinois-Chicago). In this work, the correlation between the structural and electronic properties of the grain boundaries was determined by a combination of Zcontrast imaging and EELS in the STEM. The conclusion of this work was that the oxygen anion vacancies are responsible for the varying grain-boundary properties that are observed.

S. Garofalini (Rutgers Univ.) reviewed the application of molecular-dynamics computer simulations to the study of glass surfaces and glass/glass and glass/crystal interfaces. Among the systems discussed was the lithium silicate glass/crystalline vanadia interface that has relevance in certain thin-film battery applications.

A study of the extrudation of liquid aluminosilicate from polycrystalline alumina was presented by N. Ravishankar (Univ. of Minnesota). The focus of this study was to examine the possibility of removal of an intragranular glassy phase that was introduced to generate enhanced densification through liquidphase sintering. It was shown by HRSEM that extrudation of the liquid phase out of the grain boundaries at the annealing temperature depended on the wetting behavior of the free surfaces and the wetting behavior of the boundaries.

Symposium Support: LANL and $3 M$ Co.

\section{Microstructure Engineered for Functionality}

The main emphasis of Symposium BB was the characterization and modeling of various types of microstructure such as twins, tweed, and antiphase boundaries in (mostly) martensitic and ferroelectric materials undergoing structural transformations. The key idea was to understand and then engineer these microstructures for optimizing desired functionalities, for example, larger deformation strain and stronger electromechanical effects. All three types of ferroic materials, namely ferroelectrics, ferroelastics, and ferromagnetics, were discussed.

Modeling emphasized both the phasefield and Ginzburg-Landau approaches. Strain-only Ginzburg-Landau modeling led to elastic compatibility kernels for all ferroelastic transitions ( 23 in 2D and 94 in 3D). These kernels correspond to anisotropic, long-range elastic interaction between order parameter strains that govern the microstructure. Improper ferroelastics with shuffle (e.g., NiTi) were also discussed in this context. The role of local rotations was exemplified in microstructure evolution. Electronic structure calculations and molecular-dynamics simulations were presented, with the aim of providing a way of bridging length scales. Innovative applications of shape-memory alloy strings to mimic biological functions were also illustrated. Characterization of the twin orientation mechanism, micromodulated phases, tweed, planar defects, anomalous elastic constants, and aging was highlighted for the magnetic martensite $\mathrm{Ni}_{2} \mathrm{MnGa}$.

Many novel techniques were used to characterize ferroelastic and ferroelectric domains such as second-harmonic generation, synchrotron $x$-ray, NDE, near-field scanning optical microscopy, various other scanning probes (e.g., scanning force microscopy), HRTEM, AFM, MFM, and nonlinear dielectric microscopy. Surface-induced nucleation and switching, dynamic electro-optic response (in epitaxy), nucleation and relaxation kinetics, pinning defects, domain wall migration, and mobility were also discussed.

An interesting development was the study on the "domain-material properties relationship" in domain-engineered singlecrystal PZN-PT and PMN-PT systems. By manipulating the domain structures, new symmetries can be created at the mesoscopic level, and the piezoelectric effect can be increased by almost an order of magnitude. Domain patterns also proved to be the key in ferroelectric thin films that have lattice mismatch with the substrate. It was found that the 2D cross-hatching domain patterns minimized the elastic 
energy of the whole system. Some evidence indicated that non- $180^{\circ}$ domain walls do show some movement during switching, although a majority of the domain walls are pinned by the substrate in ferroelectric thin films.

Domain microstructure has become increasingly important in materials science as it controls many physical properties of materials. It provides an intermediate size that can link the microscopic crystal structure to macroscopic properties.

Symposium Support: ARO.

\section{Novel Characterization Methods Expand Understanding of Ferroelectric Thin Films} (See MRS Proceedings Volume 655)

Understanding ferroelectric thin films through use of novel and sophisticated characterization methods was an important theme in Symposium CC. Highlights included work by K. Ito (NEC Laboratories) and A.I. Kingon (NCSU) in which SPM-based piezoresponse imaging was used to study ferroelectric fatigue in $\mathrm{Pb}(\mathrm{Zr}, \mathrm{Ti}) \mathrm{O}_{3}(\mathrm{PZT})$ films. These results suggest that loss of polarization in individual $180^{\circ}$ ferroelectric domains is an important mechanism for fatigue in these films, in addition to (or perhaps instead of) domain wall pinning. Piezoresponse imaging was also used by C.S. Ganpule and co-workers (Univ. of Maryland) to study size-dependent effects on ferroelectric switching in patterned epitaxial PZT structures. Gruverman (Sony) presented SPM results on the grain-size-dependence of ferroelectric behavior in polycrystalline films. S.V. Kalinin (Univ. of Pennsylvania) gave a detailed summary of the domain-imaging mechanisms that operate in both contact and noncontact SPM techniques. In addition to these and other contributions on domain imaging, important results on other characterization methods were presented. R. Waser (IFF, Jülich, Germany) described an important advance in quantitative analysis of switching dynamics through electrical testing, whereby the contribution of time-dependent relaxation currents can be separated from the measured current resulting directly from domain switching. S.K. Streiffer and co-workers (ANL) presented in situ characterization of $\mathrm{PbTiO}_{3}$ (PT) epitaxial MOCVD growth processes through synchrotron x-ray scattering. These experiments clearly demonstrate the effects of deposition conditions on epitaxial growth modes, and allow tentative identification of the surface reconstruction of (001) PT. I. Levin (NIST) used HRTEM and EELS to study the accommodation of nonstoichiometry in MOCVD-grown

\section{Graduate Students Receive Gold and Silver Awards}

During an awards ceremony held on November 29 at the 2000 MRS Fall Meeting, graduate student finalists received Gold and Silver Awards.

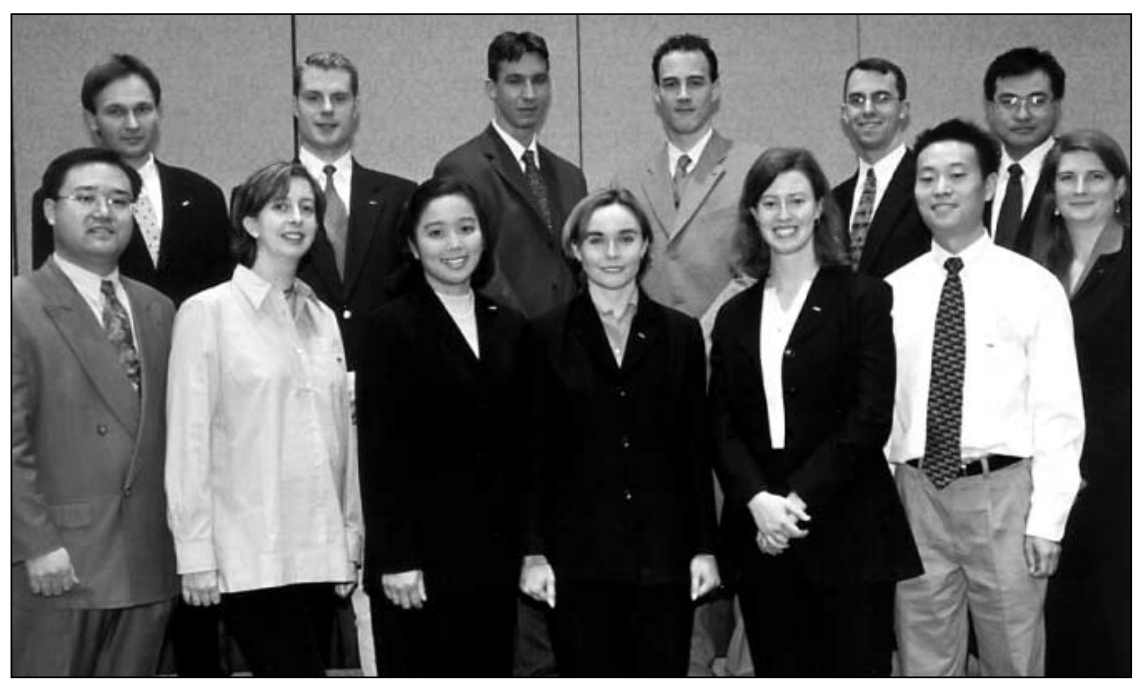

Gold Graduate Student Awards went to (first row, left to right): Jinsang Kim (MIT), Lucía Aballe Aramburu (Fritz-Haber-Inst. der Max-Planck-Gesellschaft, Germany), Wilasa Vichit-Vadakan (Princeton), Sandra Whaley (Univ. of Texas-Austin), Erin Lavik (MIT), Wei Lu (Princeton), and Michele L. Ostraat (Caltech); (second row, left to right): Thomas Rueckes (Harvard), David S. Ginger (Cambridge), Brian D. Reiss (PSU), Robert Klie (Univ. of Illinois-Chicago), John S. Lettow (MIT), and Xi-Yong Fu (Ohio State Univ.).

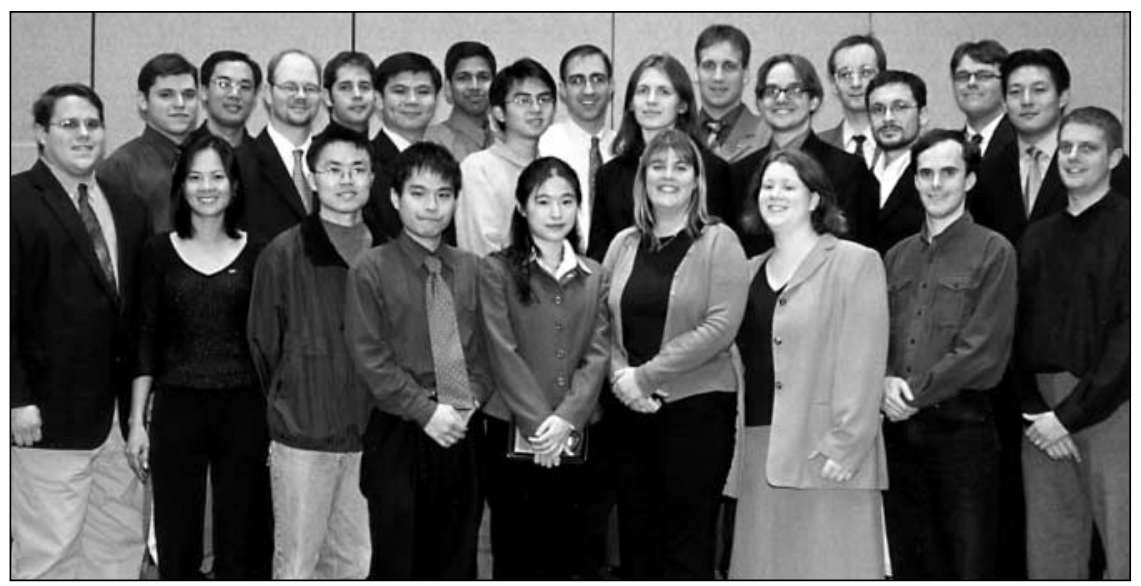

Silver Graduate Student Awards went to (first row, left to right): Tod Schneider (Kent State Univ.), Thuc-Quyen Nguyen (UCLA), Yadong Yin (Univ. of Washington),

Katsuhiko Nishiguchi (Tokyo Institute of Technology), Juan Cai (Arizona State Univ.), Abbie McLaughlin (Cambridge), Alexis C. Lewis (Johns Hopkins), Joel M. Therrien (Univ. of Illinois, Urbana-Champaign), Michael Pitcher (Univ. of MassachusettsAmherst); (second row, left to right): Alexei A. Erchak (MIT), Raymond E. Schaak (PSU), Peng Jiang (Rice Univ.), Wei Cai (MIT), Galina F. Grom (Univ. of Rochester),

Christopher F. Blanford (Univ. of Minnesota), Raúl Enrique (Univ. of Illinois, UrbanaChampaign), Edward Ahn (MIT); (third row): Kevin M. Chen (MIT), Adrian Hierro (Ohio State Univ.), Suresh R. Sriram (Univ. of Illinois, Urbana-Champaign), Byron Gates (Univ. of Washington), Olaf Pfennigstorf (Univ. of Hanover, Germany), Sergei V. Kalinin (Univ. of Pennsylvania), and Stefan Nufer (MPI für Metallforschung, Germany). Not shown are Yongmei Jin (Rutgers) and Moneesh Upmanyu (Princeton). 
polycrystalline $(\mathrm{Ba}, \mathrm{Sr}) \mathrm{TiO}_{3}(\mathrm{BST})$ films.

The challenges and prospects for integration of $\mathrm{SrBi}_{2} \mathrm{Ta}_{2} \mathrm{O}_{9}$ and PZT ferroelectric films into $\mathrm{Si}$ devices were described in invited presentations by N. Nagel (Infineon, Germany) and K.M. Lee (Samsung, Korea), respectively. Encouraging results reported by Samsung on PZT films of sub-100-nm thickness suggest the possibility of low-voltage operation of future high-density memories. Also in keeping with the theme of materials integration, J.D. Baniecki and co-workers (IBM) presented a very thorough study of hydrogen-doping effects on electronic conduction in (BST) thin-film dielectrics. Their results indicate that field-emission makes a major contribution to the enhanced leakage currents observed in hydrogen-degraded titanate thin films with Pt electrodes.

Symposium Support: Inorg Tech, Agilent Tech., ULVAC Japan, Inst. for Super Materials, Rohm Co., aixACT Systems, ATMI Ventures, Kojundo Chemical Co., and NEC Corp.

\section{Tunable RF and Microwave Device Parameters Explored}

The topics of most papers in Symposium DD were on the development of new materials, fabrication of thin films, understanding the role of stress in thin films for understanding tunability, Curie temperature, loss tangent compared with bulk materials, and RF microstructural characterization techniques. The longerterm objective for most researchers, by way of new materials, materials growth, and device design, is to obtain phase shifters that provide $>120^{\circ}$ of phase shift per $\mathrm{dB}$ insertion loss at the frequency of operation and at biasing conditions of less than $100 \mathrm{~V}$.

S. Wolf (DARPA) gave an overview of the DARPA FAME (Frequency Agile Materials for Electronics) program and showed that an antenna using paraelectric materials as the phase shifter has a reasonable radiation pattern at broadside and at the electronic scan angle of $60^{\circ}$; however, losses need to be further improved. D. Oates (Lincoln Laboratory) reported on progress of high-performance ferritetuned resonator (filter) devices at cryogenic temperatures that use high-criticaltemperature superconductors and discussed the importance of using ferro/ paraelectric materials for trimming the device.

J. Horwitz (NRL) reported the growth by pulsed laser deposition of thin films, 250-500 $\AA$, having rocking curve FWHM of 72 ", but having columnar grains and poor microwave properties. He also showed the effects that dopants like Mn,
$\mathrm{W}$, and $\mathrm{Fe}$ at the $1 \mathrm{~mol} \%$ level have on tunability and Curie temperature of thin films. Fe doping kills tunability at room temperature and severely depresses the Curie temperature. $\mathrm{Mn}$ and $\mathrm{W}$ lower the Curie temperature and broaden the dielectric response around the Curie temperature. Horwitz also discussed that, for pulsed laser deposition, the pressure of deposition influences grain size as well as in-plane and out-of-plane lattice parameters with these parameters' equivalency only at an oxygen partial pressure of deposition of about 50 mTorr. Grain size increases with oxygen partial pressure, and post-annealing in oxygen yields the highest figure of merit of the material.

Symposium Support: $A R O$ and ONR/DARPA.

\section{MEMS Moves Beyond Silicon}

\section{(See MRS Proceedings Volume 657)}

The range of topics covered in Symposium EE included metrology and tribology at small scales, processing-dependent properties, and packaging. In particular, the maturation of MEMS as a field for materials research was made evident by the presentations on new materials (proving that the field can move beyond standard silicon processing) and fatigue (demonstrating that the MEMS devices have proven sufficiently practical that reliability has become a concern).

The mechanical advantages of diamondlike carbon would be beneficial for MEMS applications, but device fabrication and stress are severe problems. A. Sumant (ANL) presented ultra-nanocrystalline diamond films, which were used to fabricate surface-micromachined devices as well as coat $\mathrm{Si}$ devices. The advantages over conventional CVD coarse-grained diamond were obvious with regard to pattern definition and conformal coatings. J. Sullivan (SNL) described amorphous diamond films. In these films, the conversion of $s p^{3}$ bonds to $s p^{2}$ bonds was shown to relieve residual stresses during annealing. This is critical for the fabrication of planar devices. M. Allen (Georgia Tech.) presented novel MEMS devices that utilized tape-cast ceramics in place of the standard Si wafers. Both a capacitive sensor and a combustion-driven magnetic electrical generator were discussed. A wide range of other materials, including $\mathrm{SiGe}, \mathrm{SiC}$, metals, and polymers, were also presented by various researchers.

While some of the attendees were skeptical that fatigue is a genuine issue with current MEMS devices, fatigue effects in MEMS specimens were undeniably demonstrated by numerous researchers both for metals and for $\mathrm{Si}$, a brittle material.

Symposium Support: Fairchild Semiconductor,
MMR Tech., Denton Vacuum LLC, Optical Micro-Machines, Cleveland Clinic Health System, TI, and Network Photonics.

\section{Facts and Fiction in Rational Materials Design Highlighted}

(See MRS Proceedings Volume 658)

Presentations in Symposium GG were directed toward rational materials design. The topics included novel synthetic methods leading to new materials, solving complex crystal structures using $4 \mathrm{D}$ crystallography, magnetic interactions in superconductors and colossal magnetoresistive solids, solid-state ionics (battery materials), microwave dielectrics, negative thermal expansion, and thermoelectric materials. The objective of many papers was to predict, understand, and control the structures of inorganic solids leading to desired properties for applications. Many new materials with potential near-term applications were reported.

Materials that offer both high electrical conductivity and high transparency to visible light have always been based on $n$-type oxides such as $\mathrm{In}_{2} \mathrm{O}_{3}: \mathrm{Sn}$. Significant new applications (e.g., transparent transistors) would be possible for $p$-type transparent conductors. J. Tate and A.W. Sleight (Oregon State) announced that their group had increased the conductivity by a factor of 200 over that reported by other groups for a $p$-type transparent conductor. Thin films of $\mathrm{CuCrO}_{2}: \mathrm{Mg}$ show a room-temperature conductivity as high as $285 \mathrm{~S} / \mathrm{cm}$ coupled with good transparency.

The cleanup of radioactive wastes demands new and better ion-exchange materials. Materials are needed with high cation exchange selectivity while remaining stable in extreme chemical, thermal, and radiological environments. These materials should also release the cation with backflushing or immobilize it into a densified ceramic matrix. T.M. Nenoff (SNL) and J.P. Parise (SUNY-Stony Brook) reported the synthesis and characterization of a new class of octahedral molecular sieves ("SOMS", $\mathrm{Na} / \mathrm{M} / \mathrm{Nb} / \mathrm{O}$ ) that are extremely selective for divalent cations (i.e., $\mathrm{Sr}^{2+}, \mathrm{Co}^{2+}, \mathrm{Pb}^{2+}$ ) and then are easily converted at $500^{\circ} \mathrm{C}$ to leach-free perovskite phases.

Symposium Support: DuPont, NSF, Intl. Journal of Inorganic Materials, Elsevier Science, Academic Press, and M. Braun.

\section{New Catalytic Materials Developed to Extend Porosity, Structure, and Composition Capabilities}

Catalysts play an important role in the oil, gas, and chemical industries, and in reducing pollution. New catalysts can lead to improvements in the selectivity and efficiency of a wide variety of 
processes. Symposium HH focused on new catalytic materials as opposed to applications of known catalysts to new reactions. Speakers covered progress in extending the key catalyst properties of porosity, structure, and composition. The synthesis of a novel porous organic catalyst with the essential features of a zeolite except high-thermal stability was reported (D. Gin, UC-Berkeley). The material is made by functionalizing and photochemically cross-linking micelles based on cheap surfactants. A pore size of $1.5 \mathrm{~nm}$ was obtained, and it was shown that the material can function as a solid, basic, shape-selective catalyst with potential application in the fine chemical or pharmaceutical industries.

New automotive catalysts were discussed by F. Witzel, (Emitec Inc.). Conventional automotive catalysts are based on ceramic honeycombs. The new catalysts are based on thin metal ribbons, which have a lower thermal mass and thereby heat up more readily, leading to a reduction of start-up emissions. The structure developed consists of a metal ribbon that is corrugated and wound into a cone. An additional refinement is to introduce horizontal ridges on the ribbon in order to tailor the gas flow for optimum catalyst performance.

Most commercial catalysts are based on oxidic materials, but non-oxides (e.g., nitrides, carbides) continue to generate interest. M. Ledoux (Univ. of Strasbourg) described the synthesis of silicon carbide nanotubes, which, following impregnation with nickel nitrate, give surprisingly active catalysts for the oxidation of hydrogen sulfide; a possible explanation is that activity may be enhanced by capillary condensation in the nanotubes.

Symposium Support: Shell Chemical Co./CRI Catalyst Co./, CRI Catalyst Co., Criterion Catalyst Co., NuVant Systems, Mittler Supply, W.L. Gore $\mathcal{E}$ Assoc., and Strem Chemicals.

\section{Phase Homogeneity and Grain Alignment Sought for $\mathrm{Bi}$ - and $\mathrm{Y}$-Based Superconductors \\ (See MRS Proceedings Volume 659)}

The thrust of many papers in Symposium II was on fabrication, characterization, and phase relations in both first-generation (bismuth-based) and second-generation (yttrium-based) superconductors. The long-term objective for most researchers was to fabricate superconductors free from secondary phases and with extremely good grain alignment. In-plane grain alignment within $5^{\circ}$ is extremely important in achieving high critical currents $\left(I_{\mathrm{c}}\right)$ in second-generation coated conductors. Second-generation HTS wires are based on coating specially prepared metal strips with HTS films using thin-film processing techniques.

S. Foltyn (LANL) presented work on the growth of thick films of YBCO with high $I_{\mathrm{C}}$ by pulsed laser deposition. The YBCO layer was deposited on flexible metallic substrates prepared by IBAD. One-meter lengths of centimeter-wide HTS tapes with $I_{c}$ values greater than 100 A (75 K, self-field) have been fabricated. On short lengths, $I_{\text {c }}$ values over $200 \mathrm{~A}$ have been achieved. Using IBAD-onmetal substrates, researchers found that as the YBCO thickness was increased to $\sim 1.5 \mu \mathrm{m}$, the product of critical current density $\left(J_{c}\right)$ and thickness increased to a maximum value of $\sim 220 \mathrm{~A} / \mathrm{cm}$. Above this thickness, however, additional YBCO produced no increase in $I_{\mathrm{c}}$. Researchers investigated this phenomenon in detail and found two problems in fabricating thicker YBCO films. First, many samples with thick YBCO had a 0.25- $\mu$ m-thick nonsuperconducting "dead layer" at the bottom interface. The second problem was that essentially no supercurrent was carried at thickness levels above 1.5-2 $\mu \mathrm{m}$. They have found that this was attributable to a transition from a dense $\mathrm{YBCO}$ microstructure to a porous, disconnected one at $\sim 1.5 \mu \mathrm{m}$. By using a $\mathrm{YBCO} /$ ceria or samarium-123/YBCO multilayer architecture, the researchers overcame the thickness effect and were able to

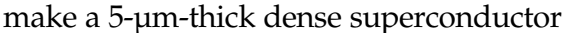
film. Foltyn reported a best result for an IBAD-on-metal substrate of $1.4 \mathrm{MA} / \mathrm{cm}^{2}$ at a thickness of $3.7 \mu \mathrm{m}$ (equivalent $I_{\mathrm{c}}$ of about $500 \mathrm{~A} / \mathrm{cm})$.

G. Daniels (Univ. of Wisconsin) presented their recent measurement of electromagnetic properties of undoped, Ca-doped YBCO, and oxygen-overdoped Ca-doped YBCO bicrystal films. The intergranular current density $\left(J_{\mathrm{b}}\right)$ measured at $44 \mathrm{~K}$ for $5^{\circ}$ [001] tilt bicrystal showed improvements for the doped samples compared with undoped YBCO. The separation between the intragranular current density $\left(J_{c}\right)$ and $J_{b}$ almost disappeared for oxygenoverdoped Ca-doped sample. The overdoped Ca-doped YBCO bicrystal behaved like a single crystal.

Symposium Support: ORNL, ANL, 3M Corp., IGC-SuperPower, and LANL.

\section{Electronic and Photonic Materials Go Organic}

(See MRS Proceedings Volume 660)

In Symposium JJ, H. Schön (Lucent) presented new studies of organic crystals as the transport medium in FETs. Refined methods of organic purification and crystal growth have blazed a trail in Nature and Science during the year 2000 in publications where extremely high mobilities are reported at low temperatures, organic crystals are used as active structures in electrically driven lasers, and where FETs are used to induce very high charge densities in organic crystals and show superconductivity at sufficiently low temperatures. The highest temperature is now $52 \mathrm{~K}$, in an organic crystal of $\mathrm{C}_{60}$ in a remarkable increase from the previous record. In the same talk, studies of FETs based on the polythiophene class of polymers reveal a granular superconducting transition observed at $2 \mathrm{~K}$.

The life track of electrons and holes recombining to a light-emitting excited state in organic LEDs is still a controversial subject. In a paper from A.S. Dhoot (Cambridge), a singlet-to-triplet ratio of 0.8 was inferred from spectroscopic studies on singlet and triplet exciton transitions within devices. This number is sufficiently different from the $1 / 3$ expected from random recombination of electron and hole, to indicate that the issue of singlet/triplet ratio is far from finished. On a related theme, J. Kido (Yamagata Univ., Japan) reported a very high efficiency triplet electrophosphorescence system, where $\operatorname{Ir}(\mathrm{PPy}) 3$ is used. The quantum yield of molecular-based OLEDs, where this triplet emitter is added in one layer, reaches $20 \%$ at low light intensity. In a polymer-based system, quantum efficiencies of $8 \%$ were reported from the same molecule, indicating that the triplet collection route may be an efficient one for extracting more light from the recombination events.

Organic and polymer LEDs are now part of industry. E. Haskal (Philips Research) described an effort in making passively addressed low-resolution monochrome displays suitable for the mobile display market. Here, ink-jet deposition is used for building the thinfilm structures emitting light. J.R. Rogers (Lucent) reported on another route to the patterning of organic electronic systems for driving smart pixels on electrophoretic screens. Here, patterning by soft lithography techniques is used to define the FETs driving the display, and thousands of transistors are defined in a single step of rubber stamping. H. Sirringhaus (Cambridge) reported similar FET logic structures produced by ink-jet printing of transistors, mostly based on the ubiquitous PEDOT polymer dispersion as a substitute for inorganic metals.

A joint session on molecular electronics and nanopatterning was held with Symposium D. The recent surge in nanotechnology in the United States was vividly illustrated in the presentation by 
T. Rueckes (Harvard) on carbon nanotube crossings as electromechanical elements for information storage.

Symposium Support: Chemipro Kasei Kaisha, Dai Nippon Printing Co., IMES Co., Matsushita Electric Works, SMK Manufacturing, Stanley Electric Co., and M. Braun.

\section{Tissue Engineering, Conductive} Polymers, and Microsphere Delivery Systems Find Role in Nervous System

Symposium OO focused on the specific structure-property-function relationships of mid- to long-term implant materials that come in contact with the nervous system. Speakers discussed the special issues that arise from the scarring process that is triggered upon implantation of materials in the brain and spinal cord and discussed various strategies to modulate this response. Materials design and fabrication and implant considerations for the following broad applications were discussed: (1) entubulation and encapsulation of neural tissue, (2) 3D scaffolds for neuraltissue engineering, and (3) drug delivery and sensing in the brain.

The importance of material geometry and its ability to work synergistically with neuronal cytoskeletal constraints for nerve guidance, along with the ability of immature astrocytes to modulate the astrocytic response to scar-inducing materials, were discussed. Novel techniques for the con- trolled incorporation of biological molecules in encapsulation and entubulation devices and the design of novel electrically conductive polymers for neural applications were presented. Synthetic, biosynthetic, and peptide-based 3D scaffolds for neural-tissue engineering and bridging of severed peripheral and central nerves were presented. In addition, the use of microsphere-based delivery systems for brain-tumor therapy was discussed, as well as the importance of modeling and controlling the diffusion characteristics of agents released in the brain by the use of multiple implants.

Symposium Support: The Whitaker Foundation and $A R O$. 